\title{
Investigating global tropical cyclone activity with a hierarchy of AGCMs: the role of model resolution
}

Article

Published Version

Strachan, J., Vidale, P. L., Hodges, K., Roberts, M. and Demory, M.-E. (2013) Investigating global tropical cyclone activity with a hierarchy of AGCMs: the role of model resolution. Journal of Climate, 26 (1). pp. 133-152. ISSN 15200442 doi: https://doi.org/10.1175/JCLI-D-12-00012.1 Available at https://centaur.reading.ac.uk/28559/

It is advisable to refer to the publisher's version if you intend to cite from the work. See Guidance on citing.

To link to this article DOI: http://dx.doi.org/10.1175/JCLI-D-12-00012.1

Publisher: American Meteorological Society

All outputs in CentAUR are protected by Intellectual Property Rights law, including copyright law. Copyright and IPR is retained by the creators or other copyright holders. Terms and conditions for use of this material are defined in the End User Agreement.

www.reading.ac.uk/centaur 
Central Archive at the University of Reading

Reading's research outputs online 


\title{
Investigating Global Tropical Cyclone Activity with a Hierarchy of AGCMs: The Role of Model Resolution
}

\author{
JANe Strachan AND Pier Luigi VidALE \\ National Centre for Atmospheric Science, Department of Meteorology, University of Reading, Reading, \\ United Kingdom \\ KEVIN HODGES \\ NERC Centre for Earth Observation, University of Reading, Reading, United Kingdom \\ MALCOLM ROBERTS \\ Met Office, Hadley Centre, Exeter, United Kingdom \\ MARIE-ESTELLE DEMORY \\ National Centre for Atmospheric Science, Department of Meteorology, University of Reading, Reading, \\ United Kingdom
}

(Manuscript received 21 December 2011, in final form 11 June 2012)

\begin{abstract}
The ability to run general circulation models (GCMs) at ever-higher horizontal resolutions has meant that tropical cyclone simulations are increasingly credible. A hierarchy of atmosphere-only GCMs, based on the Hadley Centre Global Environmental Model version 1 (HadGEM1) with horizontal resolution increasing from approximately 270 to $60 \mathrm{~km}$ at $50^{\circ} \mathrm{N}$, is used to systematically investigate the impact of spatial resolution on the simulation of global tropical cyclone activity, independent of model formulation. Tropical cyclones are extracted from ensemble simulations and reanalyses of comparable resolutions using a feature-tracking algorithm. Resolution is critical for simulating storm intensity and convergence to observed storm intensities is not achieved with the model hierarchy. Resolution is less critical for simulating the annual number of tropical cyclones and their geographical distribution, which are well captured at resolutions of $135 \mathrm{~km}$ or higher, particularly for Northern Hemisphere basins. Simulating the interannual variability of storm occurrence requires resolutions of $100 \mathrm{~km}$ or higher; however, the level of skill is basin dependent. Higher resolution GCMs are increasingly able to capture the interannual variability of the large-scale environmental conditions that contribute to tropical cyclogenesis. Different environmental factors contribute to the interannual variability of tropical cyclones in the different basins: in the North Atlantic basin the vertical wind shear, potential intensity, and low-level absolute vorticity are dominant, whereas in the North Pacific basins midlevel relative humidity and low-level absolute vorticity are dominant. Model resolution is crucial for a realistic simulation of tropical cyclone behavior, and high-resolution GCMs are found to be valuable tools for investigating the global location and frequency of tropical cyclones.
\end{abstract}

\section{Introduction}

Tropical cyclones are a topic of intense investigation. They are among the most destructive of environmental hazards, with intense, landfalling tropical cyclones often

Corresponding author address: Jane Strachan, National Centre for Atmospheric Science, Department of Meteorology, University of Reading, Reading RG6 6BB, United Kingdom.

E-mail: j.strachan@reading.ac.uk leading to significant socioeconomic impacts (Southern 1979; Pielke and Pielke 1997). It is essential to address whether coarser resolution general circulation models (GCMs) used for climate investigation and projection, as well as increasingly to estimate weather and climate risk, are capable of simulating aspects of observed tropical cyclone activity with skill. Given the processes involved, the ability of a GCM to simulate tropical cyclogenesis is a stringent test of a model's capability. 
Computational limitations place constraints on GCM experiments and a compromise must be made between resolution, spatial domain, length of simulation, size of ensemble, and physical complexity. GCMs typically have horizontal resolutions of the order of hundreds of kilometers (Solomon et al. 2007) and utilize parameterizations to represent subgrid-scale processes, such as convection. These resolutions are generally not considered sufficient to simulate tropical cyclones, and the debate continues as to whether GCMs are an appropriate tool to investigate tropical cyclone activity. It has been suggested that a resolution of $2 \mathrm{~km}$ or less is required to simulate the physical processes, such as the updraft and downdraft motions, necessary for capturing the eyewall structure and hence the maximum storm intensity (Gentry and Lackmann 2010).

Regional climate models (RCMs) can be run at resolutions allowing explicit representation of the small-scale processes operating within a tropical cyclone. However, tropical cyclone activity is governed by large-scale climate variability operating on spatial scales up to thousands of kilometers and on temporal scales up to several decades (Gray 1979). RCMs are typically driven by much lower resolution GCMs, leading to errors in the boundary forcing and a weak representation of large-scale processes such as ENSO, which are known to modulate tropical cyclone activity. Shaffrey et al. (2009) show that a move from N96 to N144 in the atmosphere (approximately $135-90 \mathrm{~km}$ ) and from $1^{\circ}$ to $13^{\circ}$ in the ocean in the High-Resolution Global Environmental Model (HiGEM) leads to a dramatic improvement in the simulation of mean tropical climate and ENSO. Resolving small-scale processes leads to an improvement in mean circulation and variability (Guilyardi et al. 2004). Roberts et al. (2009) show that improved representation of tropical instability waves in HiGEM leads to an improved ocean state and a better simulation of the atmospheric Walker circulation.

If GCMs can simulate large-scale features credibly, they can provide a modeling environment to investigate how large-scale climate variability modulates tropical cyclone behavior, potentially providing aspects of predictability. The challenge is to run GCMs at as high a resolution as possible, for multicentury integrations, to capture a sufficient range of both spatial and temporal scales. We argue that highresolution GCMs offer an opportunity to study these scale interactions and are an appropriate tool for investigating the relationship between tropical cyclones and large-scale climate variability. Insight into this relationship will improve our understanding of how tropical cyclone risks vary, from both a climate science and socioeconomic point of view.

\section{GCMs and tropical cyclone simulation}

Manabe et al. (1970) was the first to show that a lowresolution $(417 \mathrm{~km}) \mathrm{GCM}$ was able to develop cyclonelike vortices in regions where observed tropical storms develop. However, simulated storms were much larger and weaker than observed. Use of higher resolution GCMs led to increasingly more realistic storm structures (Broccoli and Manabe 1990; Haarsma et al. 1992; Bengtsson et al. 1995). Despite this, Henderson-Sellers et al. (1998) cautioned against the use of GCMs for tropical cyclone simulation, due to realism and predictive skill being limited by the coarse resolutions used at that time.

During the last decade GCM studies using 100-km resolution and higher have led to an improved representation of tropical cyclone activity, including their frequency (Kobayashi and Sugi 2004) and distribution, when compared to both observed storms and storms identified in reanalysis (Bengtsson et al. 2007). Focusing on North Atlantic hurricanes, LaRow et al. (2008), using a four-member T126 AGCM ensemble, and Zhao et al. (2009), using an AGCM ensemble with approximately $50-\mathrm{km}$ grid spacing, were also able to capture observed interannual variability with correlations of 0.78 and over 0.8 , respectively. However, the resolutions remained insufficient to capture the storm intensity.

The use of a 20-km GCM by Oouchi et al. (2006) led the way for very high-resolution GCM tropical cyclone simulations. A $20-\mathrm{km}$ resolution GCM is now being run for multidecade simulations using the Meteorological Research Institute-Japan Meteorological Agency (JMA) GCM (Murakami and Wang 2010), and multidecadal simulations of the European Centre for Medium-Range Weather Forecasts (ECMWF) Integrated Forecasting System (IFS) model have been run at up to $10-\mathrm{km}$ resolutions (Manganello et al. 2012).

This paper presents results from a hierarchy of atmosphere-only GCMs with horizontal resolutions increasing from 270 to $60 \mathrm{~km}$, using three-member ensemble integrations, to investigate the impact of resolution on the simulation of tropical cyclone activity. This range of resolutions is used to represent models that can currently be run in coupled mode for multicentury simulations to investigate the impact of long-term variability on tropical cyclone behavior; such simulations will be presented in a future paper.

Section 2 introduces the hierarchy of models and the observational and reanalysis data used to assess simulated tropical cyclone behavior. It also presents the methods used to track and assess the simulated storms. Results are presented in section 3, systematically considering simulated tropical cyclone location, frequency, 
intensity, and the impact of the large-scale environmental conditions. The results are discussed in section 4 along with concluding remarks.

\section{Models, data, and methods}

\section{a. Atmosphere-only GCM experiments}

This study utilizes a hierarchy of GCMs based on the Hadley Centre General Environmental Model version 1 (HadGEM1), a configuration of the Met Office Unified Model (fully described by Johns et al. 2006; Martin et al. 2006; Ringer et al. 2006), with horizontal resolutions ranging from N48 to N216 (see Table 1). Using a single model formulation, adjusting the grid spacing only, allows the impact of resolution on tropical cyclone simulation to be investigated independent of model formulation. Small adjustments were made to the dynamics of the model, including triggers of targeted diffusion, horizontal diffusion, and polar filtering, to allow them to run in climate mode. An additional tuning of the autoconversion parameter was required at $\mathrm{N} 216$ to balance radiation at the top of the atmosphere. Adjustments to targeted diffusion reduce the occurrence of gridpoint instabilities by increasing the local diffusion of moisture when there is convergence above a given threshold; as the resolution increases, this parameter can be increased to a higher threshold because of the improved behavior of model numerics. A change to the convective available potential energy (CAPE) time scale for N216 is justified, as highresolution models are able to sustain more energy and remove it much faster than low-resolution models. Zhao et al. (2012) find that simulated tropical cyclones may be sensitive to minor changes in model dynamics, specifically the horizontal cumulus mixing rate and divergence damping coefficient. However, our sensitivity studies indicate that the adjustments made within the model hierarchy presented in this study have minimal impact on the model simulation of tropical cyclones compared to the impact of resolution changes.

The models are run in atmosphere-only mode with prescribed monthly sea surface temperature (SST) and sea ice concentration boundary conditions from the Atmospheric Model Intercomparison Project (AMIP II) (Taylor et al. 2000), integrated for the period 1979-2002, with a three-member ensemble for each model resolution (except the N144 resolution model, for which only one simulation was available). It is important to note that the choice of SST dataset used can have an impact on tropical cyclone response, as Villarini et al. (2010) found when assessing the sensitivity of simulated North Atlantic hurricane numbers to SST datasets. This atmosphere-only GCM approach, as opposed to using
TABLE 1. The horizontal resolutions of the HadGEM1 AGCM hierarchy used in the study. Approximate resolutions $(\mathrm{km})$ are at $50^{\circ} \mathrm{N}$.

\begin{tabular}{lcccc}
\hline \hline \multicolumn{1}{c}{ Model } & Resolution & Lat & Lon & $\begin{array}{c}\text { Approximate } \\
\text { resolution }(\mathrm{km})\end{array}$ \\
\hline HadGAM-N48 & $\mathrm{N} 48$ & $2.5^{\circ}$ & $3.75^{\circ}$ & 270 \\
HadGAM & $\mathrm{N} 96$ & $1.25^{\circ}$ & $1.88^{\circ}$ & 135 \\
HiGAM & $\mathrm{N} 144$ & $0.83^{\circ}$ & $1.25^{\circ}$ & 90 \\
NUGAM & $\mathrm{N} 216$ & $0.55^{\circ}$ & 0.83 & 60 \\
\hline
\end{tabular}

fully coupled models, allows simulation results to be directly comparable to each other and to tropical cyclone observations from the same period.

The models have 38 vertical atmospheric layers extending to over $39 \mathrm{~km}$ in height. The models are configured for current climate conditions with present-day atmospheric loadings for aerosols, ozone, and greenhouse gases as described by Martin et al. (2006) and with initial conditions for 1 September 1978 derived from an analyzed state. The origin of the models in the hierarchy are summarized below. The models are all HadGEM1 (Johns et al. 2006) with different names referring to the differing resolution for historical reasons:

- N48 (HadGAM-N48): an N48 resolution version of the N96 Hadley Centre Global Atmospheric Model version 1 (HadGAM1), included to represent model resolutions typically assessed for the most recent Intergovernmental Panel on Climate Change (IPCC) Fourth Assessment Report (AR4), Solomon et al. 2007; - N96 (HadGAM): the atmospheric component of HadGEM1;

- N144 (HiGAM): the atmospheric component of HiGEM, developed from HadGEM1 through the U. K. HiGEM project (Shaffrey et al. 2009); and

- N216 (NUGAM): N216 version of HadGAM1 developed through the U. K.-Japan Climate Collaboration (UJCC).

\section{b. Observational data}

Observed tropical cyclone data have been obtained from the International Best Track Archive for Climate Stewardship (IBTrACS v03r03) (Knapp et al. 2010). IBTrACS provides a compilation of historical tropical cyclone data, merging storm information from the multiple regional observational centers into one product. It is important to remember the limitations of observational tropical cyclone data: tropical cyclone records are most comprehensive and longest in the North Atlantic basin but suffer from issues with homogeneity owing to changes in observational techniques (Landsea 2007). Records can be problematic in the northwest Pacific basin, where 
multiple observing agencies hold differing historical datasets (Ren et al. 2011), and records in other basins are regarded as poor, particularly prior to the satellite era (Landsea et al. 2006). For the 1979-2002 period of analysis, the observed database is considered sufficiently complete.

\section{c. Reanalysis data}

Observationally based analyses, or reanalyses, are used to bridge the comparison between tracked GCM data and observational data. Reanalyses provide the continuity of data of a numerical model but are constrained by vast amounts of observational data. Considering both observations and reanalyses allows a balanced approach to GCM assessment. However, reanalyses are limited by the resolution of the model used and also depend on inhomogeneous observations and how they are assimilated. The tracking algorithm (described below) is applied to the reanalysis data using the same procedure as for the GCM data, providing a direct test for the ability of the tracking methodology. Case study periods were assessed to verify the ability of the reanalyses to capture major observed tropical cyclones (not shown), providing confidence in the ability of the tracking methodology to identify and track tropical cyclones.

Three sources of reanalysis data allow further investigation of the impact of resolution alongside the GCM hierarchy. The 40-yr ECMWF Re-Analysis (ERA-40) (Uppala et al. 2005), available from mid-1957 to mid-2002, has a resolution of T159 (approximately $125 \mathrm{~km}$ ) and will be used as a comparison against the lower resolution GCMs. The ECMWF Interim ReAnalysis (ERA-Interim) is a higher resolution reanalysis (T255: approximately $80 \mathrm{~km}$ ) comparable with the higher resolution GCMs (Simmons et al. 2007). ERAInterim contains several improvements on ERA-40, including the use of four-dimensional variational data assimilation (4DVAR) and improved model physics. Data are available from 1979 until present. The Modern Era Retrospective-Analysis for Research and Applications (MERRA; Rienecker et al. 2011) is assessed as an alternative high-resolution reanalysis. MERRA, also available from 1979 until present, uses the National Aeronautics and Space Administration global data assimilation system and has a resolution of $1 / 2^{\circ}$ latitude by $2 / 3^{\circ}$ longitude.

\section{d. Tropical cyclone tracking methodology}

An objective, resolution-independent feature-tracking methodology is used to identify and track tropical cyclonelike features in the GCM output and reanalyses. This method is described fully in Hodges (1995, 1996,
1999) and Bengtsson et al. (2007). Maxima in low-level relative vorticity are used for tropical cyclone identification and tracking. Cyclone tracking algorithms adopted in past studies often use minimum sea level pressure (e.g., Hoskins and Hodges 2002) or geopotential height minima (e.g., Blender and Schubert 2000). Relative vorticity allows identification of smaller spatial scales than is possible with mean sea level pressure and hence earlier storm identification. The vorticity signatures also allow the monitoring of tropical cyclone development from very early stages as tropical easterly waves through to extratropical transition to cold core storms (if this occurs).

The tracking is applied in the region $60^{\circ} \mathrm{S}-60^{\circ} \mathrm{N}$. Data are required at a 6-hourly temporal frequency. For the Northern (Southern) Hemisphere tropical cyclone season the months May-November (October-May) are assessed. High computational and data costs justify the use of the limited seasons of analysis. For the IBTrACS data, these assessment seasons capture the main tropical cyclone activity periods in each basin, with few storms falling outside the assessment seasons. For the northwest Pacific basin, typically two to three storms per year fall outside of the assessment season. For consistency, the same periods are used when assessing both observational and reanalysis data. For initial identification and tracking, $850-\mathrm{hPa}$ relative vorticity is used on a standard spectral resolution of T42. This step ensures that the identification and tracking methodology is as resolution independent as possible. This step is kept constant for data of all resolutions, also allowing consistency in the spatial scales initially identified. Studies using different resolutions often employ resolution-dependent thresholds (e.g., Walsh et al. 2007). Questions may arise over whether initial tracking on a low-resolution grid leads to storms being missed; however, applying the tracking algorithm to a number of high-resolution test cases has proved this not to be the case, and by matching the tracks from the reanalyses with the IBTrACS data nearly every tropical cyclone can be found (not shown). Additionally, as the relative vorticity can be a very noisy field for moderate to high-resolution, using T42 helps to minimize unwanted noise.

Initially all vorticity centers with intensities greater than $0.5 \times 10^{-5} \mathrm{~s}^{-1}$ at T42 resolution are tracked (for the Southern Hemisphere, vorticity is multiplied by -1 so that potential cyclones are determined as maxima in both hemispheres; a standard criteria can then be applied globally). Vorticity center tracks with a lifetime of over two days are retained for further analysis, particularly of a potential storm's vertical structure. For the retained tracks to be deemed tropical cyclonelike features the following filtering criteria, applied on a finer T63 resolution grid, must be attained: 
(i) T63 relative vorticity at $850 \mathrm{hPa}$ must attain an intensity threshold $\left(\psi_{\text {int }}\right)$ of $6 \times 10^{-5} \mathrm{~s}^{-1}$;

(ii) a positive T63 vorticity center must exist at 850 , 500 , and $200 \mathrm{hPa}$, to determine a coherent vertical structure;

(iii) there must be a minimum reduction in vorticity from 850 to $200 \mathrm{hPa}$ (at T63 resolution) of $6 \times 10^{-5} \mathrm{~s}^{-1}$ $\left(\psi_{\text {diff }}\right)$, to provide evidence of a warm core;

(iv) there must be a reduction in T63 vorticity with height (checked between consecutive pressure levels); and

(v) criteria (i) to (iv) must be attained for a minimum of $n$ consecutive time steps, chosen as 4 (one day).

These are similar criteria to those used by Bengtsson et al. (2007), but with a refined warm core criteria, which reduces the identification of nontropical cyclone monsoonlike features that may be cold core in the lower troposphere and warm core aloft (Godbole 1977). Full resolution information, such as relative vorticity and $850-\mathrm{hPa}$ wind speeds, are added back onto the remaining tracks for analysis. There are no wind speed criteria in the tracking algorithm outlined, despite a wind threshold being used as a criterion in the observational data. The use of a lowlevel wind speed threshold criterion would require model output winds to be adjusted to 10-m, 10-min sustained winds; additionally the wind speed threshold would have to be adjusted for each model in the hierarchy to account for resolution changes, as outlined in Walsh et al. (2007), removing the resolution independence of the method.

The aim of the tracking approach employed is to use a set of criteria that identifies tropical cyclones at all resolutions with minimal dependence on resolution. By using a smoothed vorticity for storm identification, the impact of resolution and model differences should be reduced to a minimum.

In a typical model year, approximately 17000 high vorticity centers are identified, 4000 potential storms are vertically assessed, and less than 100 are identified as tropical cyclones, which agrees well with observations. These simple but physically based filtering steps are kept constant for all applications of the tracking algorithm. The tracking methodology employed, particularly the primary step of tracking all initial high vorticity centers on a consistent T42 grid, ensures a high degree of resolution independence and is consistent for all basins and models/reanalyses. An objective, resolution-independent storm identification approach is essential when comparing results from models of different resolution and formation, as discussed in Walsh et al. (2012).

\section{Results}

The ability of the described hierarchy of GCMs to simulate different aspects of tropical cyclone behavior is assessed in comparison to observed tropical cyclone behavior, as represented by the IBTrACS observations, and to tropical cyclone behavior identified in reanalyses. The aim of this assessment is to establish aspects of tropical cyclone activity that are sensitive to GCM resolution and, if possible, to identify a resolution sufficient to investigate the impact of large-scale climate variability on tropical cyclone behavior, given the competing computational demands of simulation length, model complexity, and ensemble size. The simulations are global but, in order to keep the model assessment concise, it is necessary to focus some analysis on particular tropical cyclone basins.

\section{a. Annual number and geographical distribution of tropical cyclones}

Approximately 90 tropical cyclones are identified globally each year, with a standard deviation of 10 . However, we currently do not have a theory that predicts even the order of magnitude of this number of storms (Emanuel 2008).

The average annual tropical cyclone numbers for the period of analysis are summarized in Fig. 1 for the GCM ensembles, reanalyses, and IBTrACS observations. The GCM simulations reveal a clear increase in storm numbers with increasing resolution, appearing to reach a plateau at around 100 tropical cyclones for resolutions higher than $135 \mathrm{~km}$ (N96, N144, and N216), agreeing well with the observed count and the range given by the reanalyses. A relationship between model resolution and tropical cyclone formation rate, even after a resolutiondependent tropical cyclone detection threshold has been applied, is in agreement with the findings of Walsh et al. (2012). This pattern of increase with resolution holds when the storms are separated into the tropical cyclone basins (shown in Fig. 2). In the Northern Hemisphere basins, at higher resolutions the GCMs have a good agreement with observations, although the GCMs simulate slightly less storms than observed in the North Atlantic and northeast Pacific basins. The GCMs have a more even split in storm numbers between hemispheres than observed, with more than twice as many storms identified in the South Pacific. An overly strong and extended SPCZ in the model (which can be seen in Fig. 6 of Shaffrey et al. 2009) may contribute to this high storm count in the models. The higher resolution ERA-Interim and MERRA reanalyses capture the observed hemispheric split, and their South Pacific basin counts agree well with IBTrACS. However, both models and reanalyses capture tropical cyclonelike features off the coast of South America, where only one recorded storm currently exists: Catarina in 2004. Possible reasons for this are discussed below. The ERA-40 overproduces tropical cyclone 

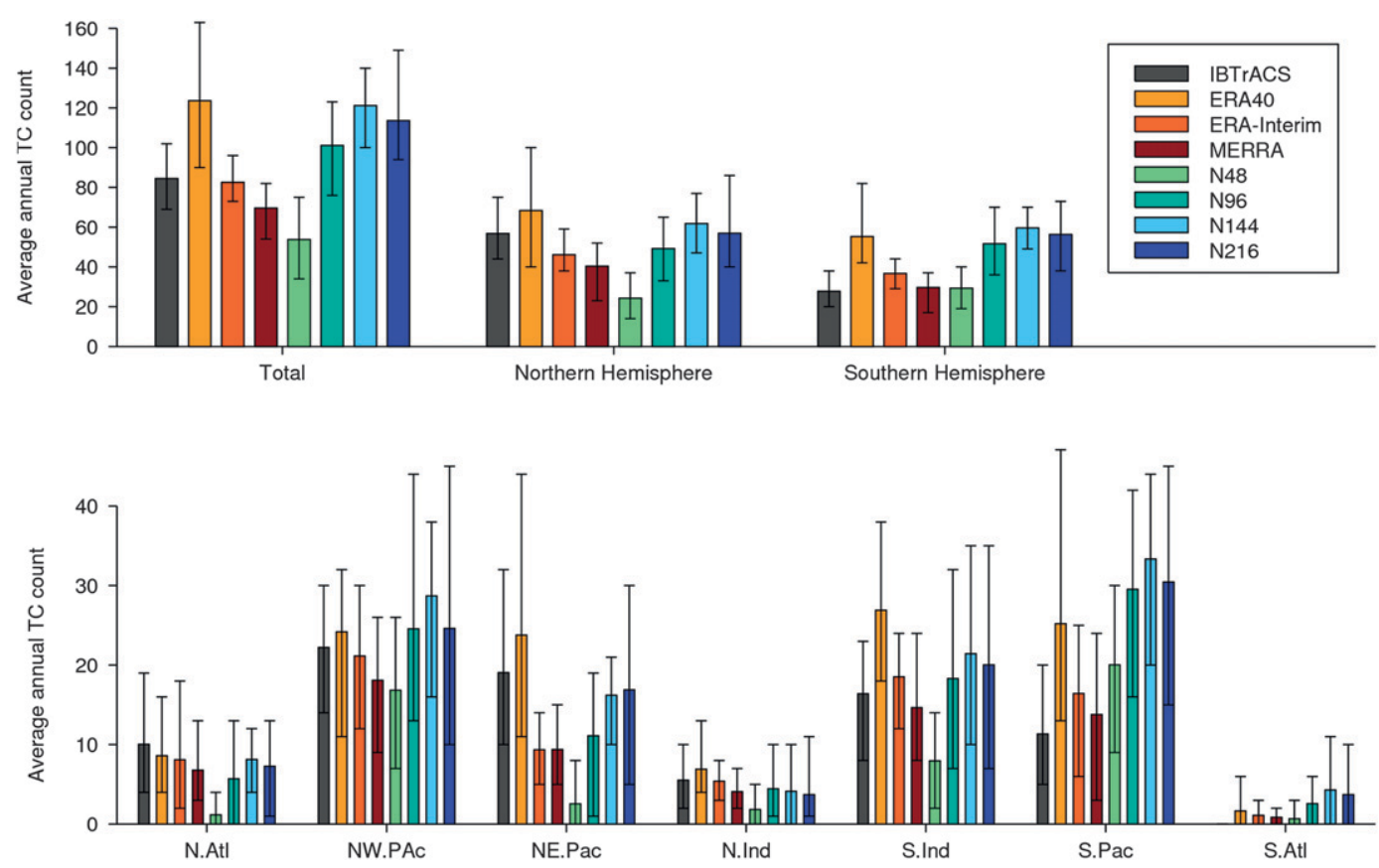

FIG. 1. Average annual (1979-2002) tropical cyclone numbers from IBTrACS observations (dark gray); ERA-40, ERA-Interim, and MERRA reanalyses (yellow, orange, and red); and the hierarchy of model ensemble simulations (blues): (top) global and Northern and Southern Hemisphere counts: (bottom) counts for each tropical cyclone basin as shown in Fig. 2. Error bars show the interannual spread of the data (which is much larger than the model ensemble spread).

numbers, and the interannual range of the extracted tropical cyclones is much larger than for the higher resolution reanalyses, GCM simulations, or observations. Similar results were found by Bengtsson et al. (2007). There are known problems with ERA-40 in the tropics, due to assimilation of satellite data that resulted in excessive precipitation over the oceans (Bengtsson et al. 2004), which may impact tropical cyclone simulation. The higher resolution reanalyses produce realistic numbers of storms in the Southern Hemisphere basins but underproduce storm numbers in the Northern Hemisphere basins, particularly in the northeast Pacific. This is most likely due to issues with the reanalyses rather than the tracking algorithm. Storms are relatively small in this region and

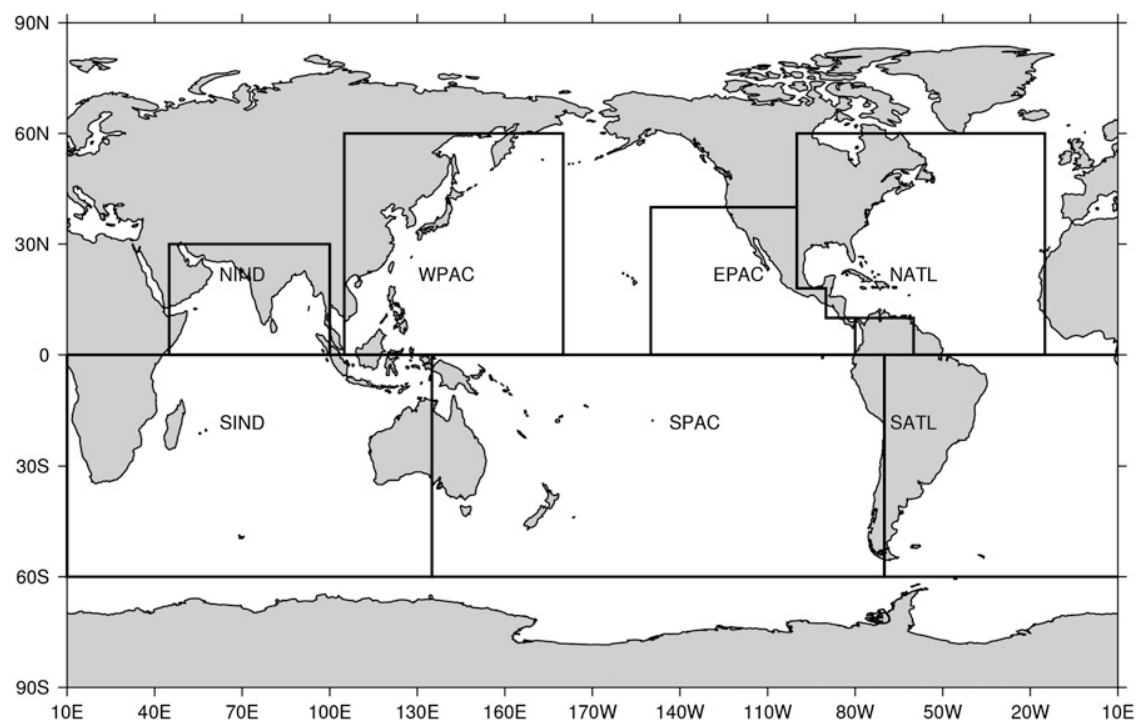

FIG. 2. Tropical cyclone basin regions, comparable to the regions used by IBTrACS. 


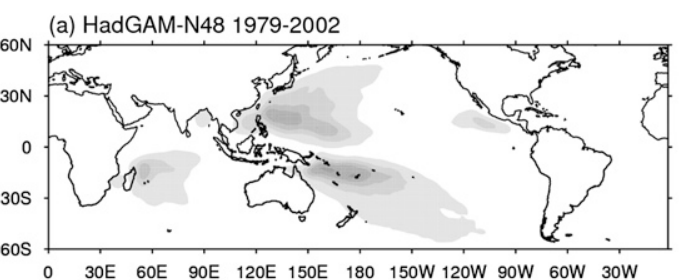

(b) HadGAM-N96 1979-2002

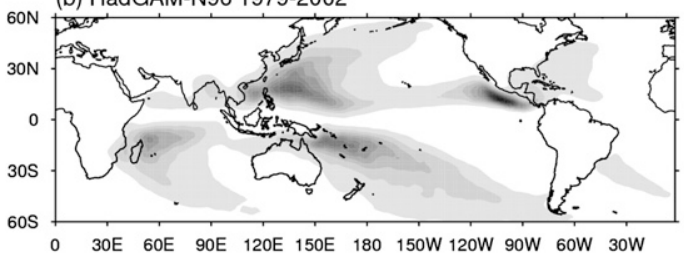

(c) HiGAM-N144 1979-2002

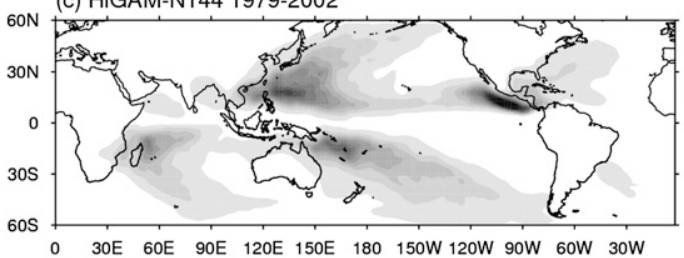

(d) NUGAM-N216 1979-2002
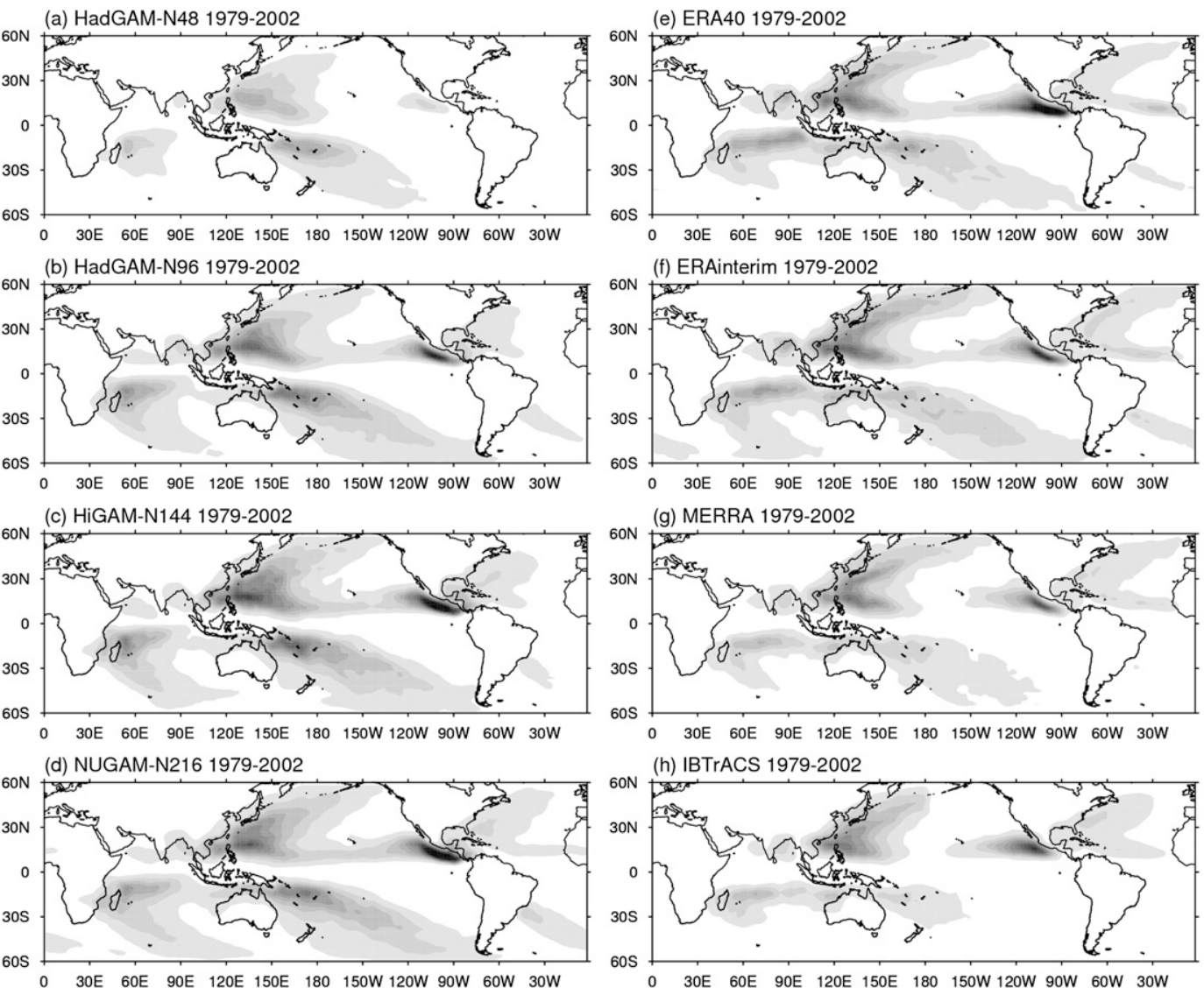

(f) ERAinterim 1979-2002

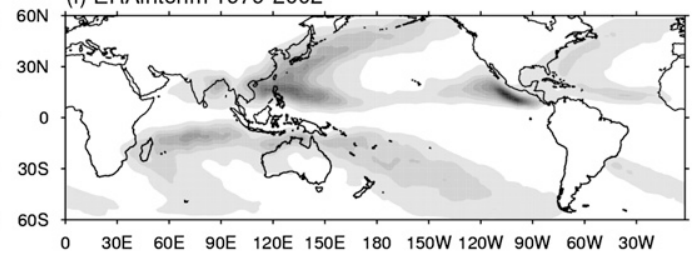

(g) MERRA 1979-2002

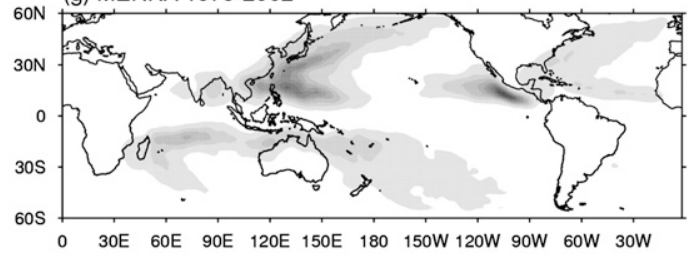

(h) IBTrACS 1979-2002

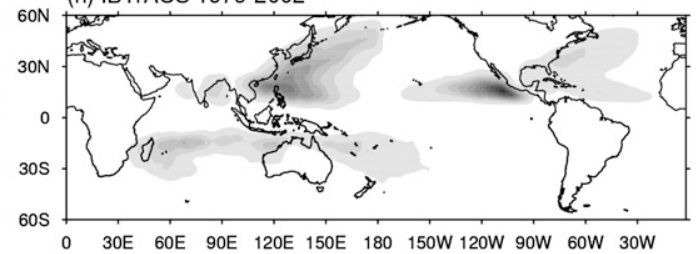

0. 30E 60 E $90 \mathrm{E} \quad 120 \mathrm{E} \quad 150 \mathrm{E} \quad 180$ 150W $120 \mathrm{~W}$ 90W $60 \mathrm{~W}$ 30W

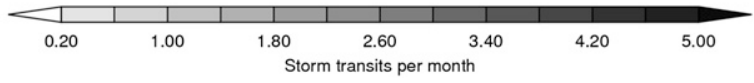

FIG. 3. Tropical cyclone track density (transits per unit area equivalent to a $5^{\circ}$ spherical cap, or approximately $10^{6} \mathrm{~km}^{2}$ ) for (a)-(d) model hierarchy compared to (e)-(g) reanalyses and (h) IBTrACS.

the high-resolution reanalyses simulate very weak storm structures, while the lower resolution ERA-40 overproduces storms in this region, as discussed above. Additionally, problems may arise due to the influence of the orography and coastline of Central America, which may be poorly resolved (Zehnder et al. 1999).

Track density statistics help to assess the ability of the GCMs to capture the spatial distribution of storms. Tropical cyclones from the analysis period are composited and monthly mean storm transits per unit area (equivalent to a $5^{\circ}$ spherical cap, or approximately $10^{6} \mathrm{~km}^{2}$ ) are calculated. Figure 3 shows the track density distributions for the GCM ensembles, alongside corresponding track densities from the reanalyses and IBTrACS observations. Figures 4 and 5 show the equivalent genesis and lysis density distributions.

The tracking algorithm follows the full life cycle of the storm from early vorticity maxima identification, the simulated genesis (when the initial T42 vorticity maxima threshold is achieved), through to lysis (when the T42 vorticity maxima threshold is no longer achieved), which may extend into the high latitudes as the storm makes extratropical transition. This tracking method leads to differences in the density distributions for the model and reanalysis storms when compared to IBTrACS, for which data are only recorded while storms are classified as tropical cyclones. The differences can be seen in the lysis density plots (Fig. 5) where GCM and reanalysis storm lysis are identified later than for IBTrACS, particularly for west Pacific storms, with lysis spreading out into higher latitudes. We have not attempted to "cut" the tracked storms to match the observed storm tracks, as this would remove valuable information about the tracked storm's origin and subsequent life cycle. Despite this difference, the model spatial distribution of storms, particularly for the higher resolutions, is in very good agreement with the observed tropical cyclone distribution. Resolution has the largest impact when moving from N48 
(a) HadGAM-N48 1979-2002

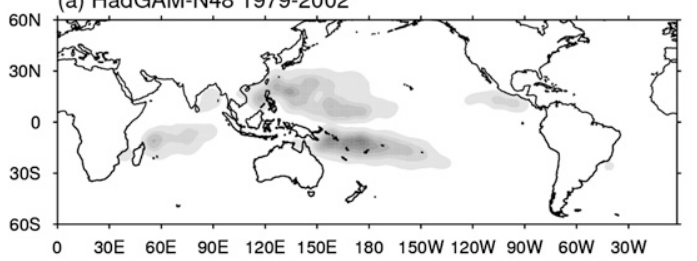

(b) HadGAM-N96 1979-2002

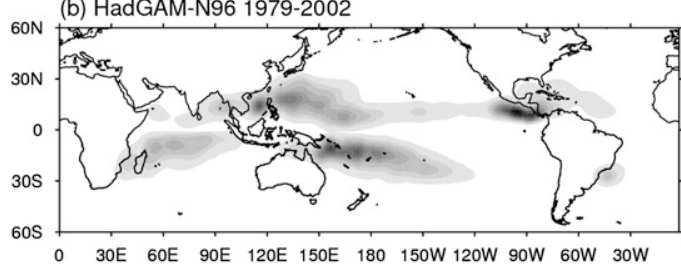

(c) HiGAM-N144 1979-2002

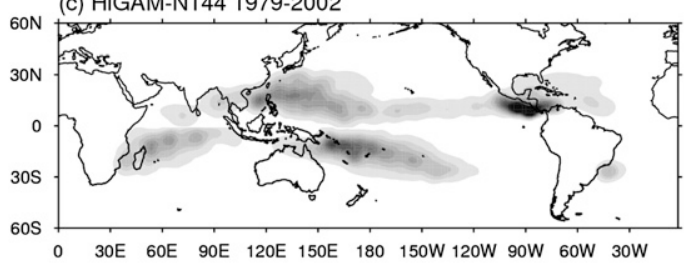

(d) NUGAM-N216 1979-2002

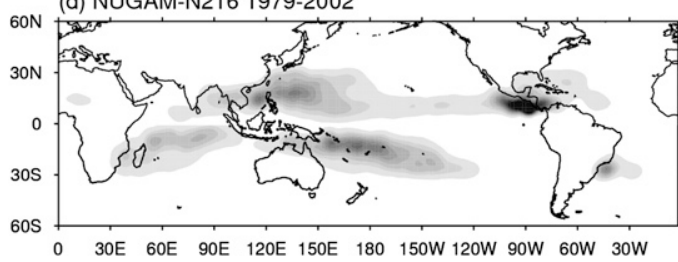

(e) ERA40 1979-2002

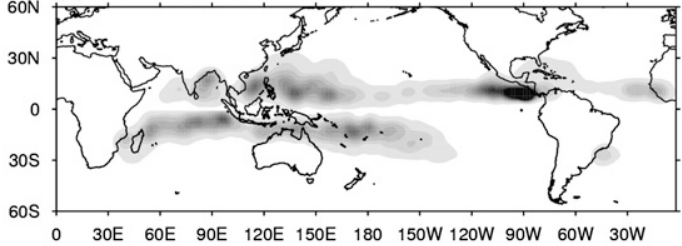

(f) ERAinterim 1979-2002

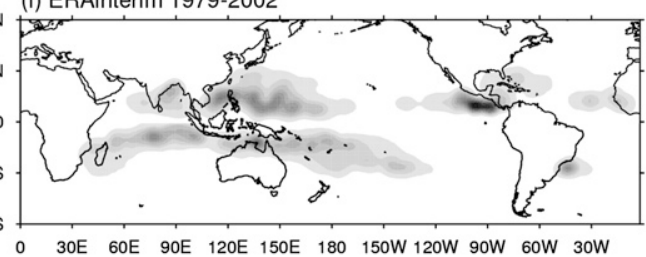

(g) MERRA 1979-2002

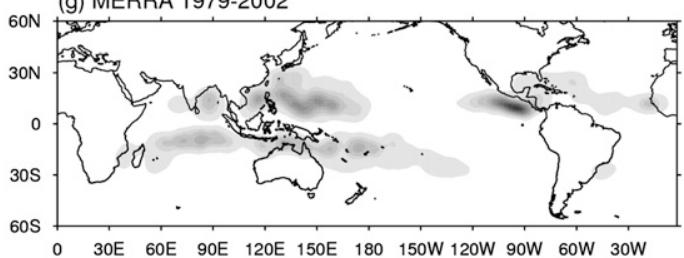

(h) IBTrACS 1979-2002

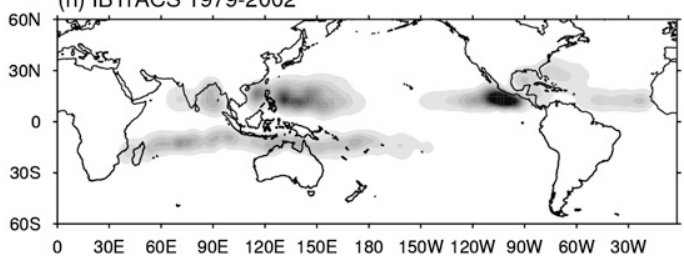

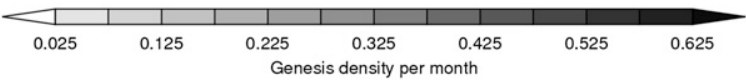

FIG. 4. As in Fig. 3 but for genesis density (genesis per unit area equivalent to a $5^{\circ}$ spherical cap, or approximately $10^{6} \mathrm{~km}^{2}$ ).

to N96, and saturation appears to be reached for the N144 and N216 GCMs.

The North Atlantic and northeast Pacific basins are the most sensitive to resolution, with only N144 and N216 GCMs giving adequate distributions [this result agrees with past studies, e.g., Bengtsson et al. (2007)]. The number and distribution of simulated North Atlantic basin tropical cyclones are in good agreement with IBTrACS at N216 resolution, but with track density slightly displaced away from the U.S. East Coast. The eastern Atlantic is an important area for storm development, given that about $85 \%$ of observed North Atlantic hurricanes that develop into major hurricanes originate from atmospheric disturbances (easterly waves) over northern Africa (Landsea 1993). The lower resolution GCMs show little genesis here compared to observations and reanalyses (Fig. 4); however, the N216 resolution captures early genesis over West Africa (Fig. 4d). The Geophysical Fluid Dynamics Laboratory (GFDL) High-Resolution Atmospheric Model
(HiRAM), in contrast, has a lack of genesis in the Gulf of Mexico (Zhao et al. 2009), whereas the ECHAM5 model underproduces storms in the mid-Atlantic (Bengtsson et al. 2007).

The east Pacific storms have a concentrated distribution, simulated well at higher resolution. Simulated storms are identified slightly earlier in their life cycle than in observations and track along the coast too closely, possible due to the influence of the modeled orography and coastline. The lysis density reveals that reanalysis and IBTrACS tropical cyclones travel farther into the central Pacific before lysis, while the models have an excessive concentration of storm dissipation around the Pacific coast of Mexico, indicating more short-lived tropical cyclones, possibly due to a difference in the large-scale steering flow.

In the west Pacific, the most active basin, tropical cyclone simulations are less sensitive to resolution, and even the N48 GCM reproduces a reasonable spatial distribution. However, simulated storms are shifted 


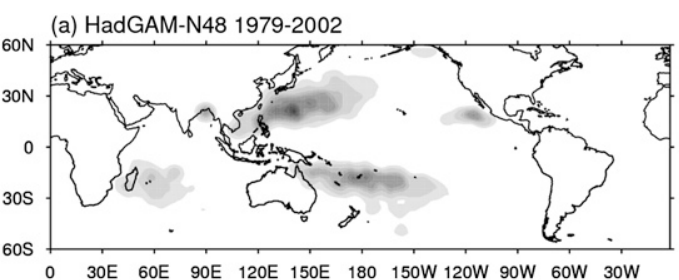

(b) HadGAM-N96 1979-2002

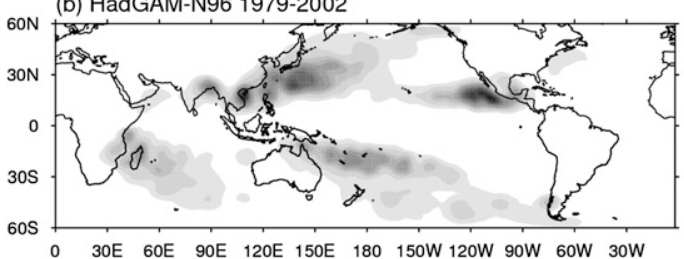

(c) HiGAM-N144 1979-2002

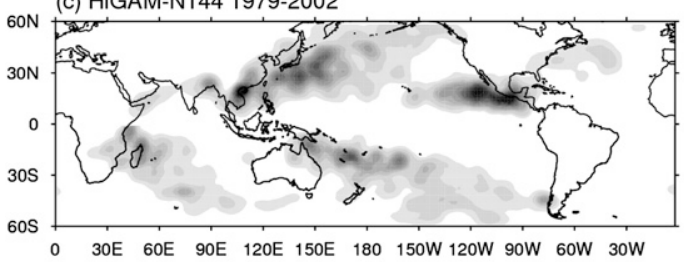

(d) NUGAM-N216 1979-2002
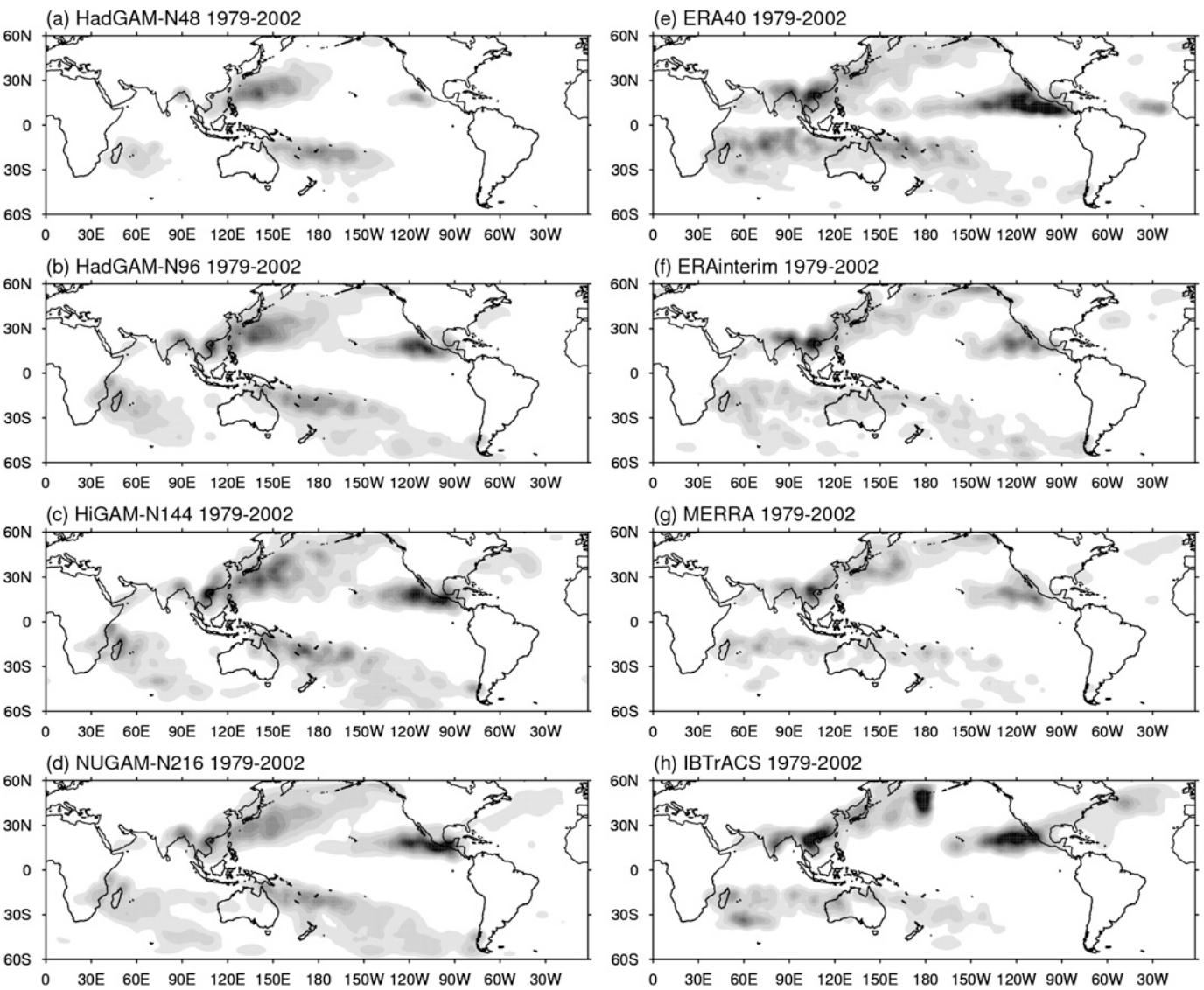

(f) ERAinterim 1979-2002

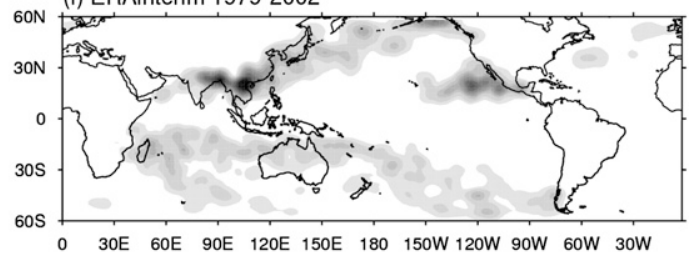

(g) MERRA 1979-2002

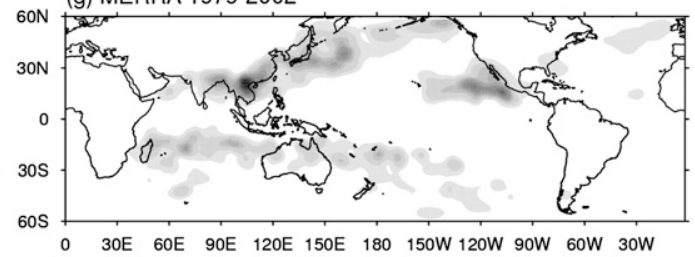

(h) IBTrACS 1979-2002

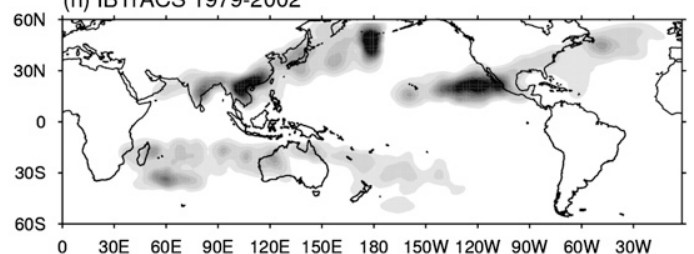

O. $30 \mathrm{E}$ 60E $90 \mathrm{E}$ 120E $150 \mathrm{E} \quad 180 \quad 150 \mathrm{~W} 120 \mathrm{~W}$ 90W $60 \mathrm{~W}$ 30W

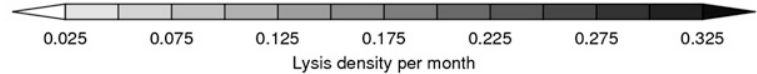

FIG. 5. As in Fig. 3 but for lysis density (lysis per unit area equivalent to a $5^{\circ}$ spherical cap, or approximately $10^{6} \mathrm{~km}^{2}$ ).

slightly away from the coast, with few appearing to make landfall. At higher resolutions the track density agrees well with observations and reanalyses, although, unlike observations and the high-resolution reanalyses, genesis occurs from the central Pacific and, as resolution increases, lysis stretches farther into high latitudes.

In the north Indian Ocean, there are fewer simulated storms than observed. Genesis occurs to the east of the basin, close to Thailand, rather than in the center of the Bay of Bengal, and storms do not travel west and affect the Indian subcontinent.

In the Southern Hemisphere, for the higher resolution GCMs, there is an excessive number of storms, particularly in the South Pacific basin. The tracking algorithm may be identifying tropical cyclones that are not being recorded in observations: that is, in areas that fall outside the remit of the tropical cyclone regional specialized meteorological centers (RSMCs) or tropical cyclone warning centers (TCWCs), where there is no chance of landfall, such as around the South Pacific convergence zone (SPCZ) and the southern Atlantic. This is supported by the IBTrACS data for the South Pacific, which only appear to be recorded up to $240^{\circ} \mathrm{E}$, the edge of the RSMC's area of responsibility. It is also possible that the tracking algorithm identifies more storms with a tropical cyclonelike (warm core) structure than would be identified by a more subjective operational criterion. This may reveal a slight deficiency in the tracking algorithm or the operational criteria thresholds, which have been tuned for the Northern Hemisphere.

Overall, the numbers and geographical distribution of tropical cyclones are sufficiently simulated by the GCMs for resolutions higher than $135 \mathrm{~km}$, a threshold that agrees well the findings of Murakami and Sugi (2010). Even for lower resolutions tropical cyclones generally only occur in the observed locations.

\section{b. Tropical cyclone frequency: Interannual variability}

Although the atmosphere-only GCM approach has its limitations [such as no two-way interaction between 


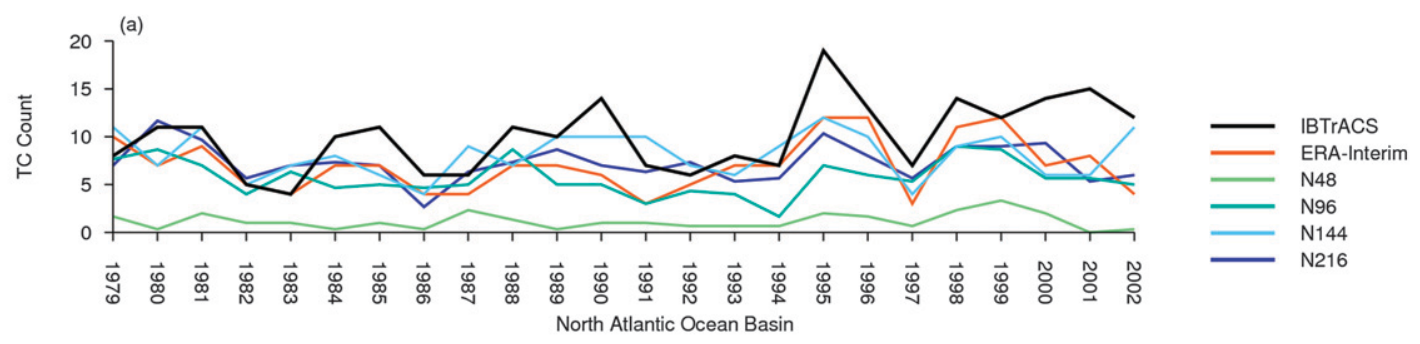

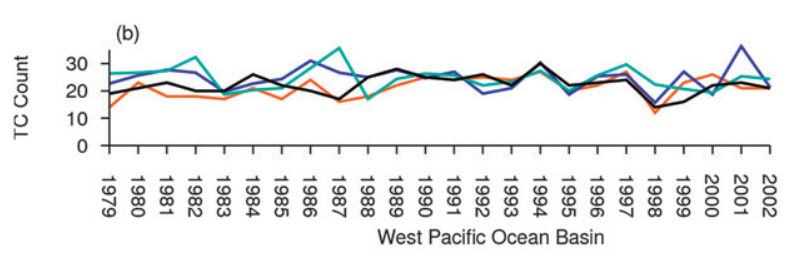
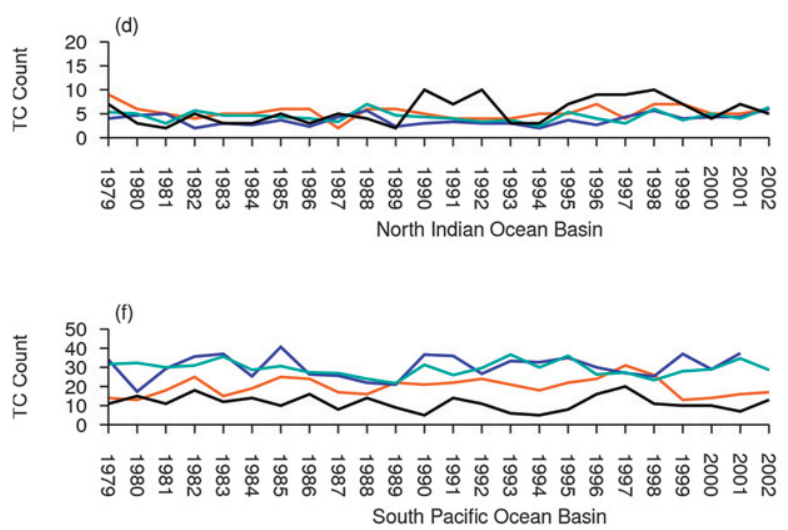
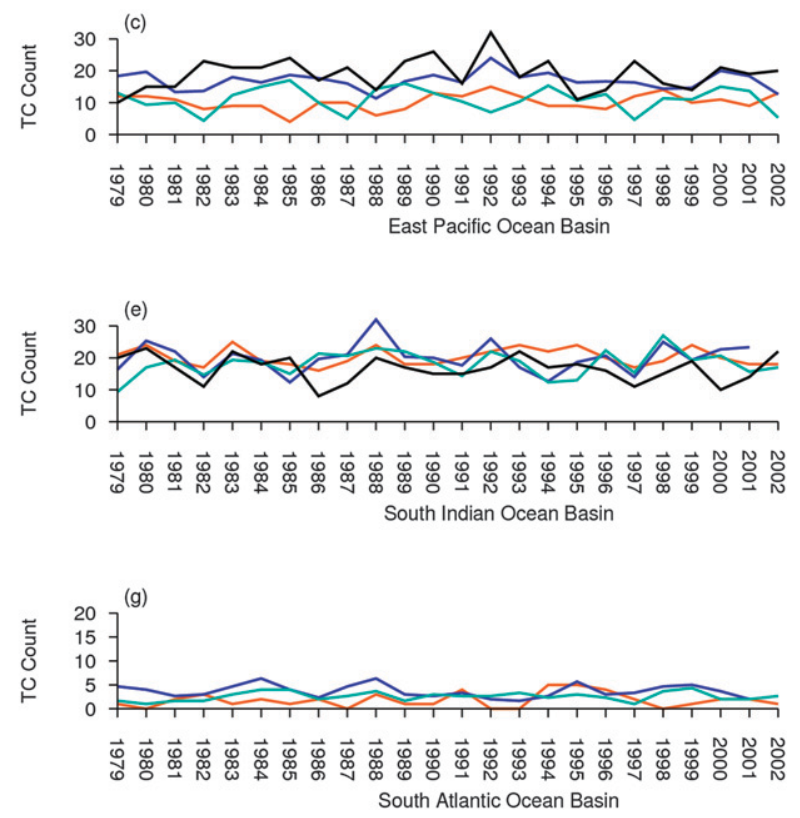

FIG. 6. Tropical cyclone interannual variability for storm basins shown in Fig. 2, showing annually varying GCM ensemble-mean (for N216 and N96) simulated tropical cyclone numbers compared against those for ERA-Interim and IBTrACS. Note that for clarity the N144 and N48 model resolutions are only shown for the North Atlantic.

the ocean and atmosphere, as described in Zhao et al. (2009)], it allows the frequency of the simulated storms to be directly compared to those from observations and reanalyses. Annual storm count time series for the period of analysis for each tropical cyclone basin (Fig. 2) are shown in Fig. 6. These show the N216 and N96 GCM ensemble-mean interannual variability alongside the IBTrACS and ERA-Interim interannual variability. Figure 6a, for the North Atlantic, also shows the N48 and N144 resolutions.

Focusing on the North Atlantic basin, Fig. 7a shows the interannual variability of tropical cyclone numbers for the IBTrACS and the reanalyses. There is good correspondence between these time series, which is confirmed by the correlations for the individual reanalyses between 0.61 and 0.78 . Higher resolution reanalyses perform better than ERA-40, showing that sufficient resolution is required to simulate interannual variability. Examining the N216 GCM ensemble compared to IBTrACS and ERA-Interim reanalyses in Fig. 7b indicates that the high-resolution GCM is able to capture interannual variability quite successfully, capturing many of the time series maxima and minima. The correlation of the N216 GCM ensemble mean with IBTrACS is 0.67 (with an ensemble spread from 0.40 to 0.74 ), similar to the correlations for the comparable resolution reanalyses, revealing significant skill when the model is simply forced with observed SST boundary conditions but is otherwise free running. The plots showing the N216, N96, and N48 GCM ensemble interannual variabilities (Figs. $7 b, c, d$ respectively), as well as corresponding correlation statistics (Table 2), clearly show a general decrease in agreement with observations as GCM resolution is reduced.

One aspect of the North Atlantic interannual variability that is not captured in the simulated tropical cyclones, at any of the resolutions, is the increasing trend observed during the assessed period (Holland and Webster 2007). Further modeling studies are being 

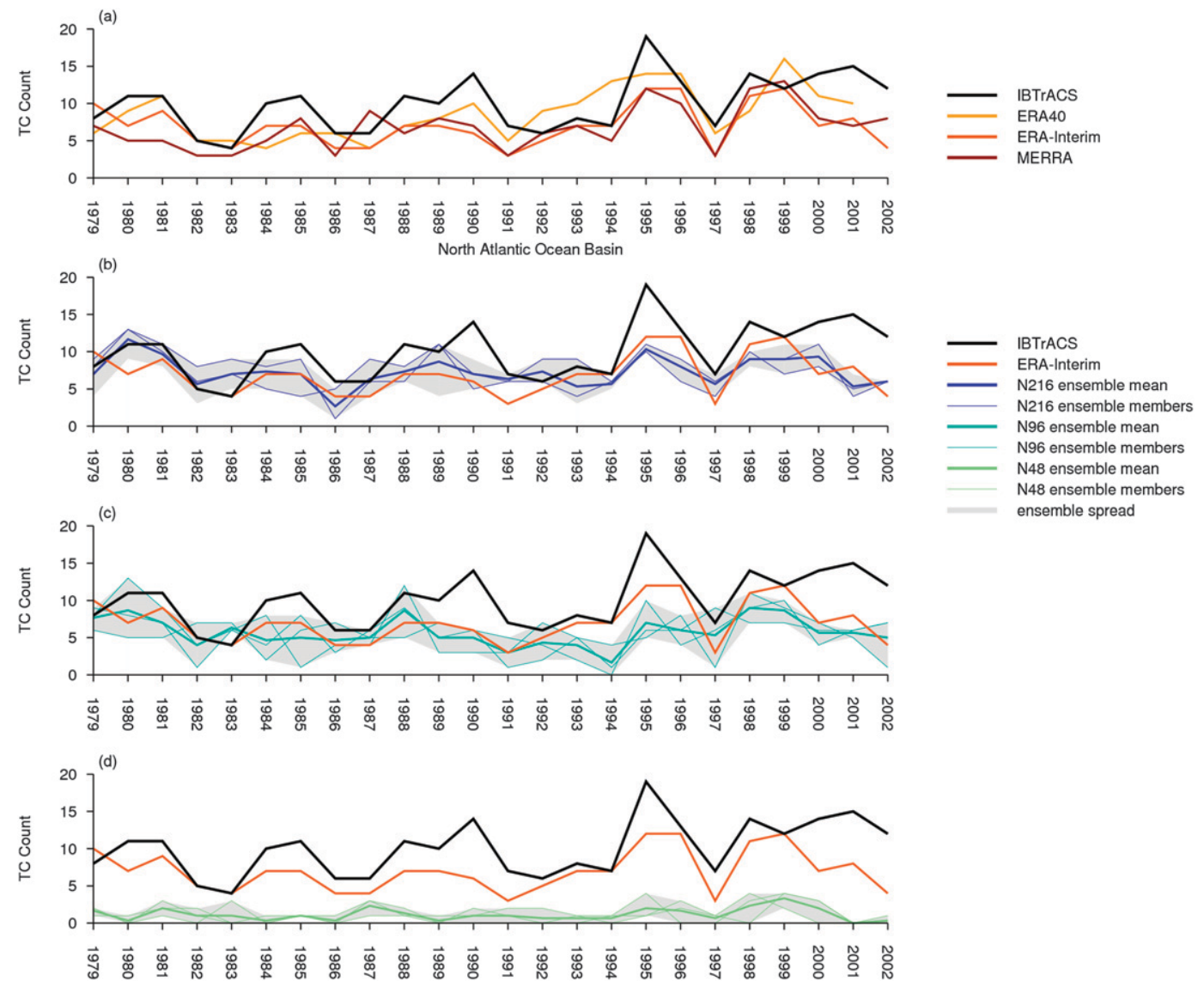

FIG. 7. Tropical cyclone interannual variability for North Atlantic basin for (a) reanalysis products; (b) the N216 GCM ensemble; (c) the N96 GCM ensemble; and (d) the N48 GCM ensemble. For (b)-(d) thick lines show the GCM ensemble mean, thin lines show the individual ensemble members, and the gray envelope shows the ensemble spread.

undertaken to examine whether it is possible to reproduce this background trend through imposed anthropogenic forcing or through better representation of the stratosphere and, hence, outflow temperature (Emanuel 2010). However, the simulated trend may significantly depend on the SST used, as has been found with the GFDL HiRAM model; the increasing trend was simulated, consistent with observations, if the Hadley
Centre Global Sea Ice and Sea Surface Temperature (HadISST) SSTs were used, but little trend was simulated when using Reynolds SSTs (M. Zhao 2012, personal communication). Additionally, Landsea (2007) suggests that some of the observed trend could represent a bias due to the introduction of new observing systems, such as the Quick Scatterometer (QuikSCAT), and identification of shorter-lived storms.

TABLE 2. Correlations between IBTrACS and the model and reanalysis interannual variability for the period 1980-2000. The N48, N96, and N216 columns show the correlation for the three-member model ensemble simulations. Bold values indicate statistical significance at the $95 \%$ confidence level, assuming the individual years are temporally independent.

\begin{tabular}{lcccrrrr}
\hline \multicolumn{1}{c}{ Basin } & ERA-40 & ERA-Interim & MERRA & N48 & N96 & N144 & N216 \\
\hline North Atlantic & $\mathbf{0 . 6 1}$ & $\mathbf{0 . 7 8}$ & $\mathbf{0 . 7 2}$ & $\mathbf{0 . 4 5}$ & $\mathbf{0 . 5 2}$ & $\mathbf{0 . 5 4}$ \\
West Pacific & 0.39 & $\mathbf{0 . 5 7}$ & $\mathbf{0 . 5 2}$ & 0.29 & -0.09 & 0.39 & $\mathbf{0 . 6 7}$ \\
East Pacific & 0.34 & 0.18 & 0.29 & -0.31 & -0.07 & $\mathbf{0 . 4 9}$ \\
North Indian Ocean & 0.41 & 0.05 & 0.18 & -0.01 & -0.01 & 0.26 \\
South Indian Ocean & 0.00 & $\mathbf{0 . 7 5}$ & $\mathbf{0 . 4 5}$ & -0.39 & -0.15 & -0.01 & 0.14 \\
South Pacific & $\mathbf{0 . 6 5}$ & 0.34 & $\mathbf{0 . 4 6}$ & -0.19 & -0.27 & 0.14 & -0.28 \\
\hline
\end{tabular}


In the northwest Pacific, the tropical cyclone interannual variability for the higher resolution reanalyses is in good agreement with the observed interannual variability (Fig. 6b). The higher resolution GCMs (N216 and N144) show weak positive correlations. Lower correlations than in the Atlantic may to be due to the SSTs (providing the interannually varying signal to the models) in the west Pacific having less of an effect on tropical cyclone numbers. It has been suggested in both empirical (Gray 1979) and modeling studies (Bengtsson et al. 1995) that a threshold SST of $26^{\circ} \mathrm{C}$ is a necessary (but not sufficient) condition for tropical cyclone development. In the Atlantic region, where SSTs are around this critical threshold, the number of tropical cyclones is sensitive to the underlying SSTs. In contrast in the west Pacific, where SSTs are significantly warmer than this threshold SST, changes in SSTs (e.g., variability associated with ENSO) are more likely to lead to a shift in region of tropical cyclone activity and the storm intensity (Camargo and Sobel 2005). Figure $6 \mathrm{~b}$ also shows that for the west Pacific basin the model resolution is not so crucial for the number of storms per year, unlike in the North Atlantic basin.

The interannual variability of the simulated storms in the northeast Pacific (Fig. 6c) is in good agreement with observations for the highest resolution GCMs (N144 and N216). The lower resolution models do not capture the variability. For this basin the reanalyses are less able to capture the observed interannual variability than for the North Atlantic and northwest Pacific basins, with correlations of around 0.3.

Both models and reanalyses fail to capture the observed interannual variability in the north Indian Ocean basin (Fig. 6d). This could be due to identification of monsoon depressions; however, the revised warm core identification should limit this. In the Southern Hemisphere basins (Figs. 6e-g) the GCMs are unable to simulate the interannual variability regardless of resolution, although the reanalyses have some skill. This suggests that SST may not be a dominant environmental variable in these basins. Analysis of other key largescale environmental factors, such as sensitivity to vertical wind shear (Gray 1984; Vitart and Stockdale 2001), are considered in section $3 \mathrm{~d}$.

It would be useful to assess intra-annual and longerterm modes of variability against the observations. However, the lack of consistency of the observational record outside the satellite era limits the assessment period (Landsea 2007).

\section{c. Tropical cyclone intensity}

To assess the impact of resolution on the intensity of the simulated tropical cyclones, the distributions of

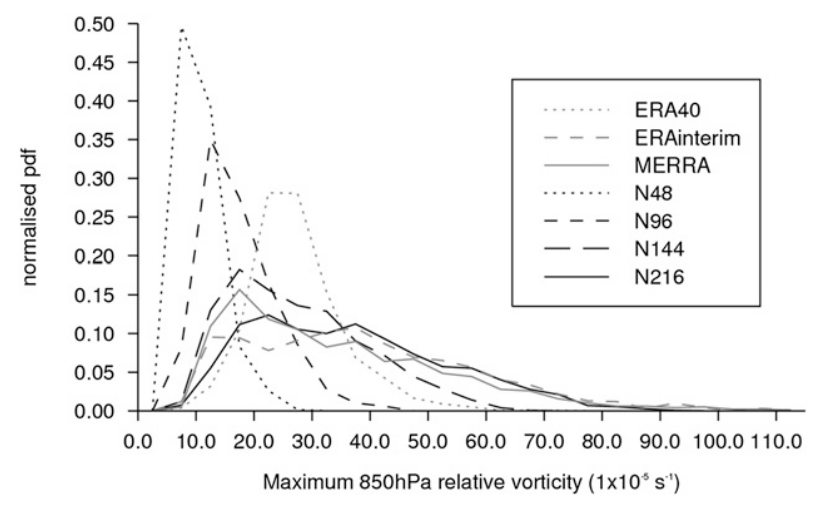

FIG. 8. Normalized distributions of Northern Hemisphere extracted storm maximum intensities in terms of maximum $850-\mathrm{hPa}$ relative vorticity from the hierarchy of GCM resolutions and reanalyses (bin widths are $5 \times 10^{-5} \mathrm{~s}^{-1}$ ).

maximum intensities from the storms identified in each GCM are compared against the distributions of intensities from the storms extracted from the reanalyses. There are three ways to compare intensity: surface wind, pressure, and $850-\mathrm{hPa}$ relative vorticity. A choice is made to assess tropical cyclone intensity in terms of storm maximum $850-\mathrm{hPa}$ relative vorticity, as this parameter is much more sensitive to resolution changes and therefore highlights differences in intensity distribution more clearly than with the wind speed and mean sea level pressure. There are limitations regarding the use of the $850-\mathrm{hPa}$ relative vorticity, as it does not allow direct comparison with available observed wind intensities, which would be possible through the use of mean sea level pressure or 10-m, 10-min sustained winds. The main disadvantage of not comparing directly against the "truth" in terms of observations is that it is difficult to determine how deficient the simulated storm intensities are. The main concern regarding the use of model output wind speeds to assess the distribution of maximum intensities against observations is that the winds are not directly comparable in terms of vertical level, temporal sampling, and resolution. These issues are discussed in detail by Walsh et al. (2007).

The distributions of maximum intensities in terms of maximum relative vorticity, shown in Fig. 8a, reveal a shift toward tropical cyclones of higher intensity is clear for higher resolutions for both the GCMs and reanalyses. The distributions shift in terms of the mean maximum intensities and the tail of maximum intensities (indicating the most intense storms), with distributions both flattening and lengthening with resolution. The intensity distributions for the GCMs and reanalyses of similar resolutions are in excellent agreement, with the MERRA and ERA-Interim almost overlaying the NUGAM intensity distribution. It is difficult to indicate 

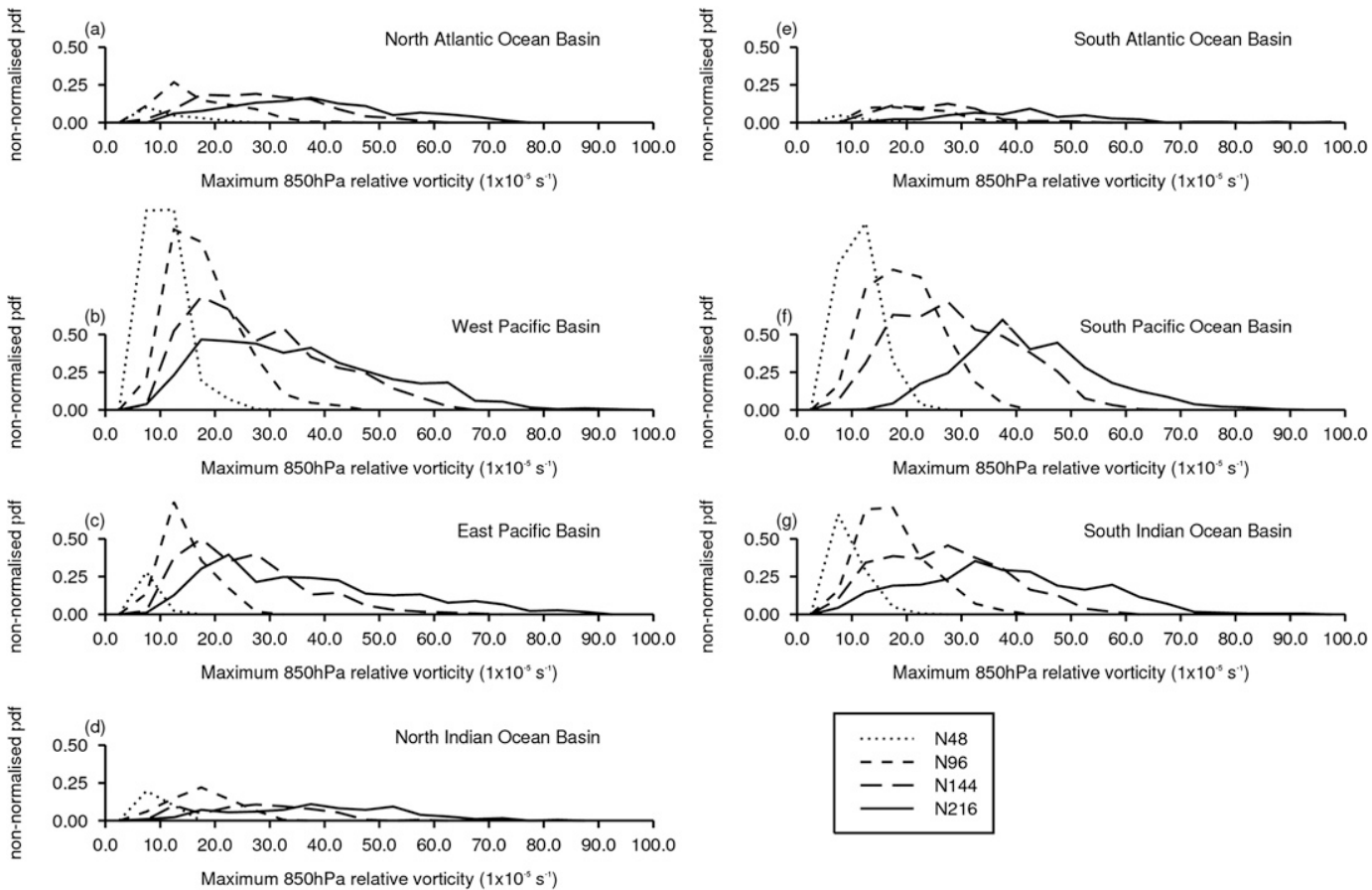

FIG. 9. Nonnormalized distributions of maximum intensities for the various tropical cyclone basins, in terms of $850-\mathrm{hPa}$ relative vorticity for the GCM resolution hierarchy. Bin widths are $5 \times 10^{-5} \mathrm{~s}^{-1}$.

the truth in this plot, because of the use of relative vorticity, but it allows better comparison between the resolutions and reanalyses. However, the maximum 850-hPa wind speed distributions (not shown) indicate that the resolutions used in this investigation are too coarse to be able to simulate the observed intensities.

Figure 9 shows the intensity distributions by tropical cyclone basin, providing a more detailed view of the regional impact of resolution. These plots are nonnormalized to show how the number of storms changes in each basin with resolution. In all basins there is a shift to more intense storms seen in both the peak of the distribution and the tail. In the North Atlantic basin there is a large increase in storm numbers from N48 to N96 resolution and from N96 to N144. However, from N144 to N216 the change is in the shape of the intensity distribution rather than the number of storms, suggesting that above a threshold resolution is less critical for the simulation of the number of tropical cyclones but remains crucial for simulating storm intensity. A similar pattern of change is also seen in the east Pacific. In most of the other basins, there is little increase in storm number above N96 resolution with most of the change occurring in the intensities of the storms. This finding is consistent with the discussion in section 3b: where the SSTs are generally above the $26^{\circ} \mathrm{C}$ threshold, storm count is less sensitive to changes in SST.

\section{d. Tropical cyclones and large-scale environmental conditions}

The following analysis focuses on assessing the influence of large-scale environmental factors known to influence tropical cyclone formation and activity. The genesis potential index (GPI) motivated by the work of Gray (1979) and developed by Emanuel and Nolan (2004) is used as the basis for this investigation, as this index is a composite of environmental variables that are believed to be physically important to tropical cyclogenesis:

(i) low-level absolute vorticity: represents the requirement of "spin" in the atmosphere to form these cyclonically rotating storms;

(ii) midtropospheric relative humidity: sufficient midlevel moistening is required as a dry middle atmosphere hinders tropical cyclone development;

(iii) vertical wind shear: as the presence of wind shear allows dry air to be advected into the moist vertical column required for tropical cyclone development; and

(iv) potential intensity (PI): a thermodynamic variable that provides a theoretical estimate of the locally achievable intensity of tropical cyclones, in terms of either maximum wind speed or minimum sea level pressure, by taking into account the sea surface 


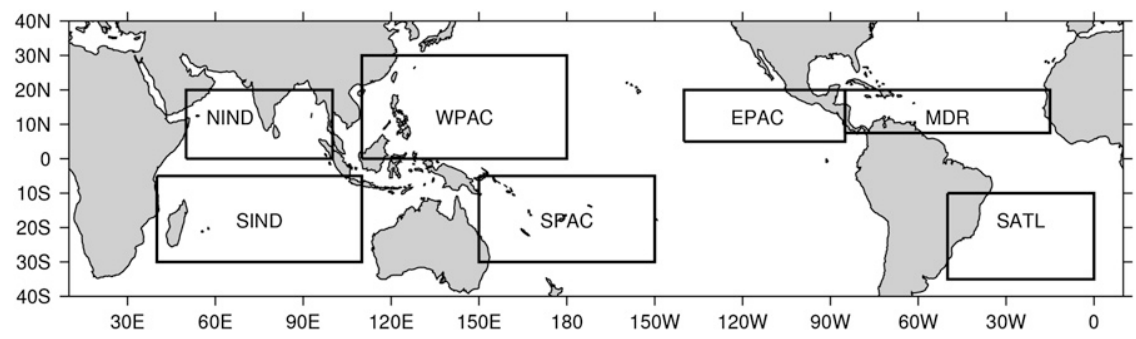

FIG. 10. Selected main development regions (MDRs) for each tropical cyclone basin.

temperature and the temperature and humidity structure of the atmosphere (Emanuel 1995; Bister and Emanuel 1998).

The index weights the various factors with functional dependences found to be appropriate to represent their relative importance in the climatology. The process used to obtain these functions was partially subjective and partially objective (based on multiple regression analysis). The GPI is defined as

$$
\mathrm{GPI} \equiv\left|10^{5} \eta\right|^{3 / 2}\left(\frac{\mathcal{H}}{50}\right)^{3}\left(\frac{V_{\text {pot }}}{70}\right)^{3}\left(1+0.1 V_{\text {shear }}\right)^{-2},
$$

where $\eta$ is the absolute vorticity at $850 \mathrm{hPa}\left(\mathrm{s}^{-1}\right), \mathcal{H}$ is the relative humidity at $700 \mathrm{hPa}(\%)$, and $V_{\text {shear }}\left(\mathrm{m} \mathrm{s}^{-1}\right)$ is the wind shear (magnitude of the vector difference between horizontal winds at 850 and $200 \mathrm{hPa}$ ). The potential intensity (PI) in terms of the maximum velocity $\left(V_{\text {pot }}, \mathrm{m} \mathrm{s}^{-1}\right)$ is defined as

$$
V_{\text {pot }}^{2} \equiv \frac{T_{s}}{T_{0}} \frac{C_{k}}{C_{D}}\left(\mathrm{CAPE}^{*}-\mathrm{CAPE}^{b}\right),
$$

where $T_{s}$ is the SST, $T_{0}$ is the mean outflow temperature (at the level of neutral buoyancy), $C_{k}$ is the exchange coefficient of enthalpy, and $C_{D}$ is the drag coefficient. The CAPE is the vertical integral of parcel buoyancy as a function of parcel temperature, pressure, specific humidity, and vertical profiles of virtual temperature: CAPE* is the value of CAPE for an air parcel that has been lifted from saturation level at the sea surface temperature and pressure, while $\mathrm{CAPE}^{b}$ is the value of CAPE for boundary layer air. Both are evaluated at the radius of maximum winds.

The GPI and the individual environmental variables are calculated for the main development regions (MDRs) of the tropical cyclone basins as shown in Fig. 10. Monthly mean values of the GPI are averaged across the basin MDRs to construct a climatology of the seasonal cycles of tropical cyclone activity in each basin (Fig. 11). All resolutions in the GCM hierarchy are able to capture the seasonal cycle of GPI, agreeing well with the reanalyses, and capturing, for example, the double peak in tropical cyclone activity in the north Indian Ocean basin around the monsoon season. The relationship between tropical cyclone activity and the monsoon season using the GPI is investigated in more detail in Evan and Camargo (2011). However, the amplitude of the seasonal cycle in the Northern Hemisphere basins is smaller in the models in comparison to the reanalyses. When N216 GCM monthly mean tropical cyclone numbers are superimposed onto these plots (gray outlined histograms), the seasonal cycle of simulated tropical cyclone activity is in good agreement with the GPI seasonal cycle, particularly in the North Pacific and North Atlantic basins, agreeing with the findings of Camargo et al. (2007). This gives confidence that the GCMs are able to simulate the climatology of environmental conditions important for tropical cyclogenesis. Comparing GPI values against tropical cyclone numbers suggests that the N216 model is slightly underproducing tropical cyclones in the North Atlantic and north Indian Ocean basins in response to the environmental conditions, whereas it is overproducing tropical cyclones in the South Pacific and south Indian Ocean basins. Similar analysis undertaken by Walsh et al. (2012) finds that coarser-resolution models simulate the GPI better than they simulate formation of tropical cyclones directly.

Tropical cyclone season means [July-October (JASO) for Northern Hemisphere basins; DecemberMarch (DJFM) for the Southern Hemisphere] of GPI and its constituent environmental variables are calculated and averaged over the basin MDRs. This is done to isolate relative contributions from the environmental factors toward interannual variability of tropical cyclones in the different basins and allows an assessment to be made of how well the models simulate the environmental conditions for tropical cyclone activity, compared to that seen in reanalyses. Scatterplots and associated correlations are used to determine statistical relationships between the large-scale environmental variables and annual tropical cyclone numbers for the different basins. 

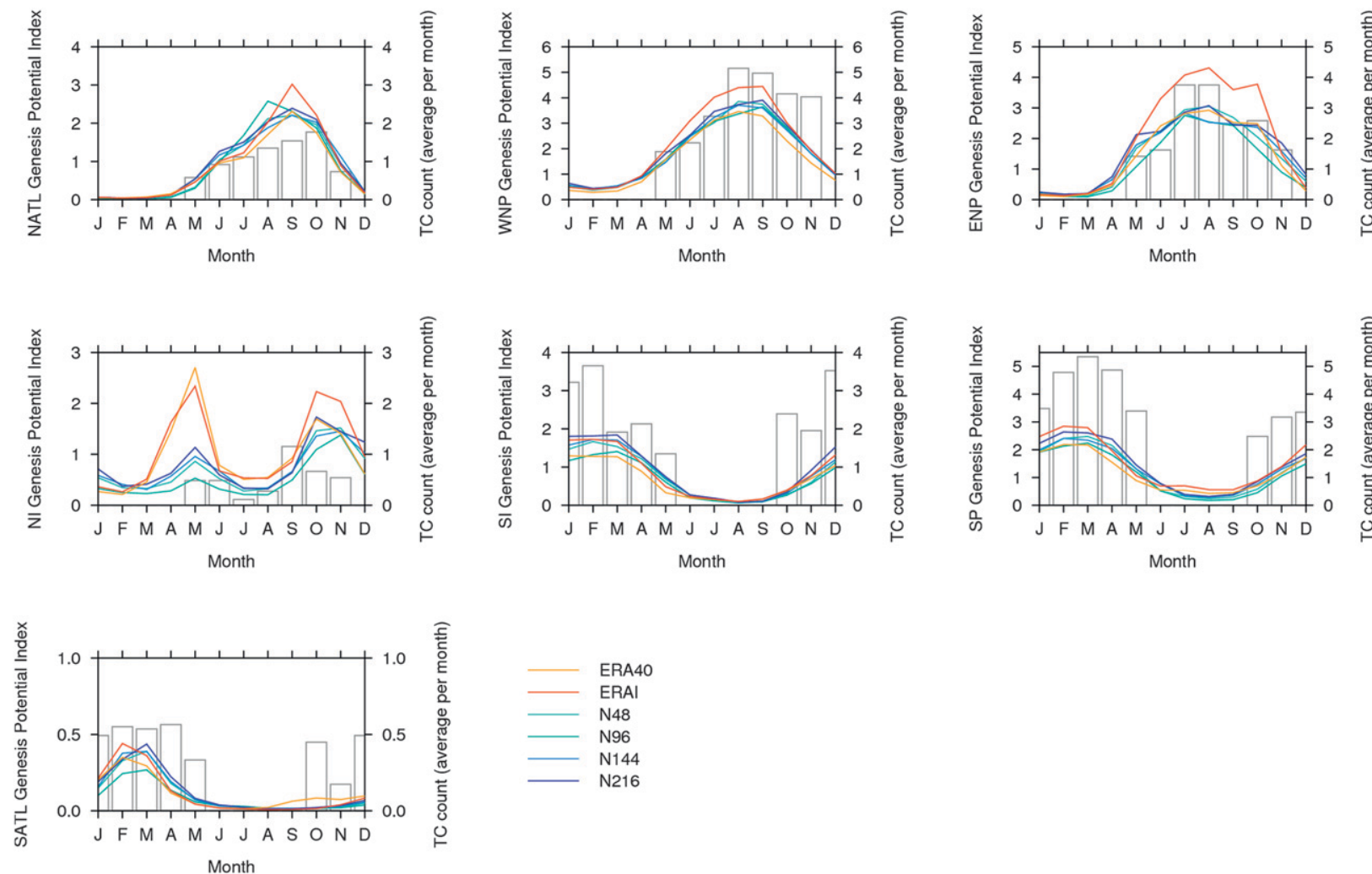

FIG. 11. Seasonal cycle climatology of GPI for the MDRs, defined in Fig. 10, and the monthly-mean tropical cyclone count in that basin. Results from the model hierarchy and the ECMWF reanalyses are shown. The monthly-mean tropical cyclone numbers from N216 ensemble simulations are superimposed on the GPI seasonal cycle using gray outlined histograms.

Plots are only included for the North Atlantic and northwest Pacific basins.

In the North Atlantic basin (Fig. 12), where the GCMs and reanalyses are able to capture the observed interannual variability of storms, ERA-Interim and ERA40 have good correlations between JASO GPI and tropical cyclone count ( 0.56 and 0.38 , respectively). Relatively good correlations are also found for the higher resolution GCMs. Absolute vorticity has significant interannual variability correlation with storm count in ERA-40 (0.55), ERA-Interim (0.54), and the N216 GCM (0.48). Relative humidity at $700 \mathrm{hPa}$ does not reveal any strong correlations with storm count, apart from in ERA-40 (0.35); a negative correlation for the N216 GCM may be contributing to the lower overall GPI correlation. The vertical wind shear has strong interannual variability with high vertical wind shear leading to reduced storm count in both GCMs and reanalyses. Correlations are stronger for the reanalyses than for the GCMs $(-0.77,-0.52$, and -0.43 for ERAInterim, ERA-40, and the N216 GCM, respectively). The PI component also has a strong interannual correlation with storm count in ERA-Interim (0.70). This positive correlation is seen for the GCMs, with N216 and
N96 giving correlations of 0.37 and 0.39 respectively. This is lower than for ERA-Interim, suggesting that the GCMs struggle to capture the interannual variability of the atmospheric temperature and humidity structure as represented by the PI.

In the Atlantic basin the ENSO has an impact on the interannual variation of tropical storm activity. How well the ENSO signal will be transmitted to an atmosphereonly GCM and to the corresponding tropical cyclone variability will depend on how the model tropical cyclone basin responds to the prescribed SSTs, which carry the interannually varying ENSO signal. In the Pacific Ocean basin, the ENSO signal is felt directly through the SSTs, whereas in the Atlantic basin the ENSO variability has a strong influence through wind shear modulation. The reanalyses are likely to have a stronger correlation with interannual variability associated with ENSO because they are assimilating other variables such as winds, which will also carry an ENSO signal. This may be why stronger interannual correlations exist between atmospheric variables and storm count for the reanalyses. The ENSO signal will be partially transmitted through the prescribed SSTs, which the models will respond to through largescale atmospheric response. 


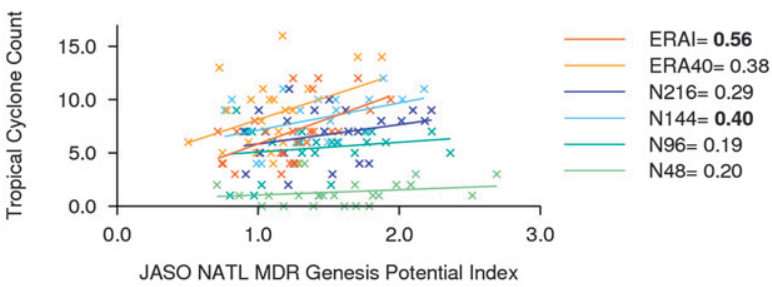

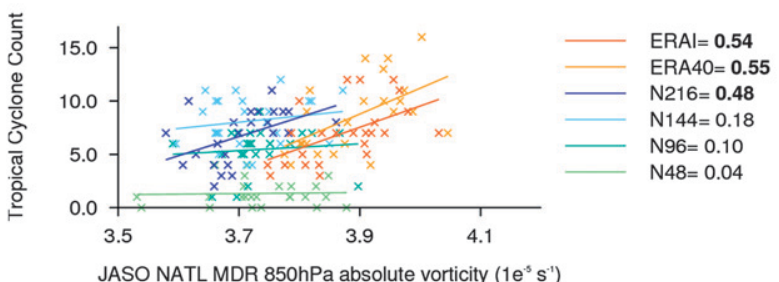

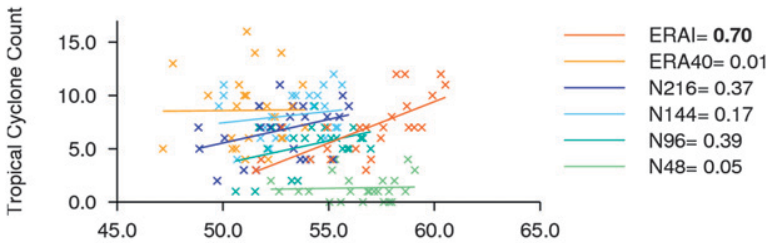

JASO NATL MDR 850hPa Potential Intensity (Maximum Velocity $\mathrm{m} \mathrm{s}$ )
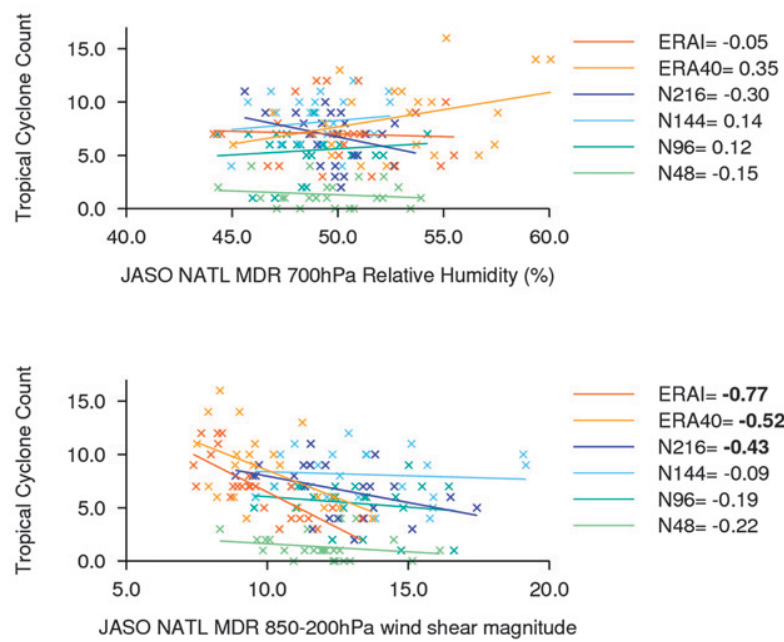

FIG. 12. North Atlantic annual tropical cyclone frequency vs JASO main development region (as defined in Fig. 10) means of GPI and component variables of GPI for the N216, N144, N96, and N48 model resolutions and ERA-40; and ERA-Interim reanalyses. Lines of best fit and corresponding correlations are also shown. Bold values indicate statistical significance at the $95 \%$ confidence level, assuming the individual years are temporally independent.

For the northwest Pacific basin (Fig. 13), where the interannual variability of storms agrees well between IBTrACS and the higher resolution GCMs and reanalyses, a correlation between JASO GPI and tropical cyclone count is only seen for ERA-Interim. When the constituent large-scale environment components of GPI are assessed an interesting picture emerges, which may explain the low correlation between annual storm count and GPI in the GCMs. The PI component of the GCMs and ERA-40 have negative correlations with storm count $(-0.31$ to -0.42 in the GCMs and -0.46 for ERA40). This relationship is consistent with findings of the study by Zhao and Held (2012) in which the AGCM simulated hurricane response to coupled GCM (CGCM) generated future SST anomalies (see their Fig. 4). The finding is supported by Chan and Liu (2004), who found a lack of relationship between local SST and northwest Pacific tropical cyclone activity and a negative correlation between the ratio of typhoons to tropical cyclones and SST in the region $5^{\circ}-30^{\circ} \mathrm{N}, 120^{\circ} \mathrm{E}-180^{\circ}$, very similar to the chosen MDR. SST change in the northwest Pacific often leads to a geographical shift in storms, rather than a change in number of storms, as the area of warm SSTs expands/contracts (Chia and Ropelewski 2002). The weak/negative correlations of PI with tropical cyclone count may be capturing this response. Additionally, JASO vertical wind shear has positive correlations with storm count for the GCMs (from 0.30 to 0.57 ) becoming stronger with resolution, opposite to what would be expected-a response that again is found in the study by Zhao and Held (2012). It was thought that this may be due to the chosen MDR region, which includes regions of varying vertical wind shear; however, smallerfocused regions returned similar results (not shown). Aiyyer and Thorncroft (2011) find that, although interannual variability of shear over the northwestern Pacific is found to be closely tied to that of ENSO, which may not be captured in the AGCM approach used in this study, shear was found to have no coherent relationship with typical cyclone activity in the northwest Pacific on interannual time scales. Midlevel relative humidity has strong correlations with the storm count for the higher resolution GCMs and ERA-Interim. There are also strong correlations with $850-\mathrm{hPa}$ absolute vorticity, increasing with GCM resolution (0.31-0.73), also seen in the reanalyses.

For the basins that are not shown, different environmental factors emerge as being important depending on the basin. In the northeast Pacific, relative humidity 

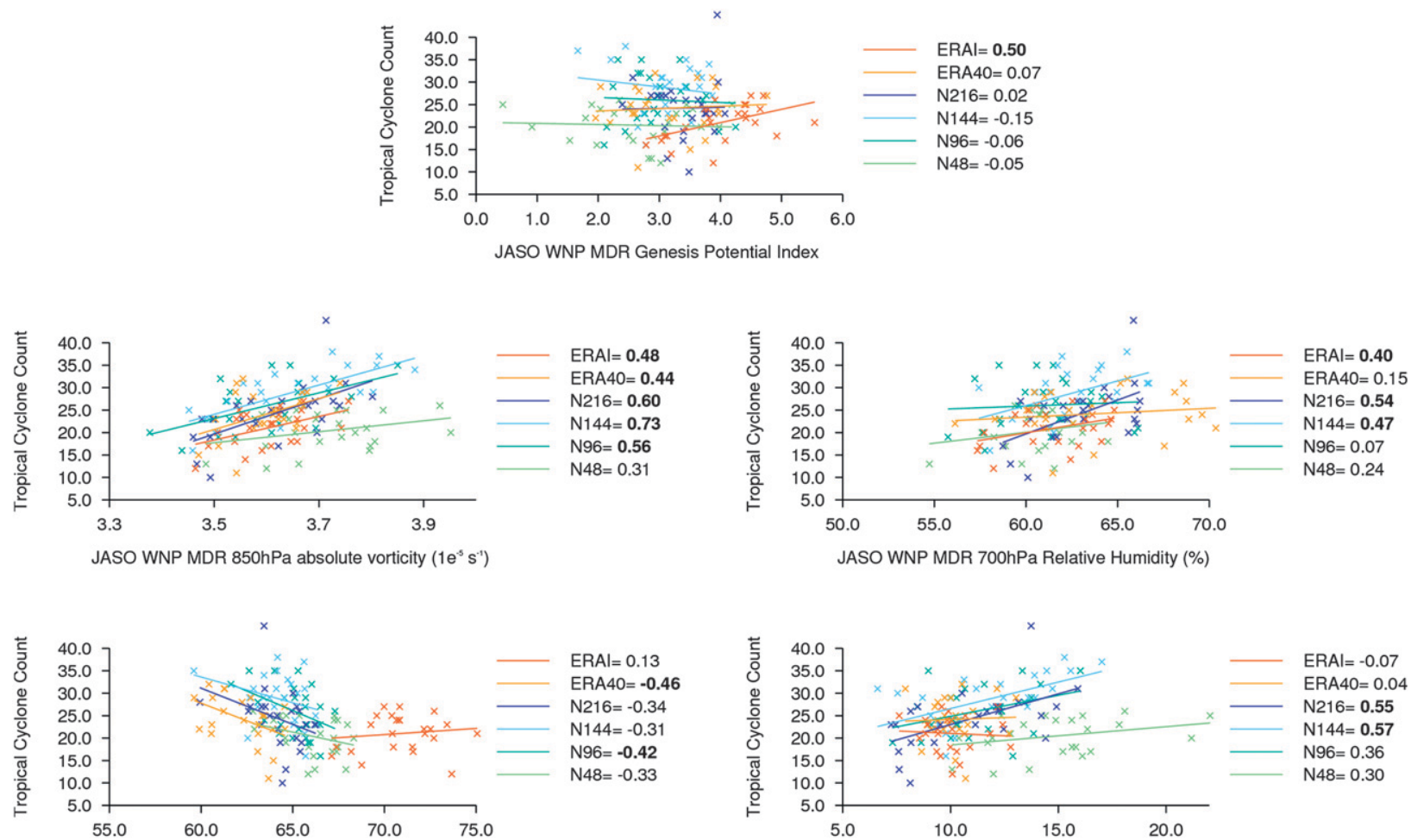

JASO WNP MDR $850 \mathrm{hPa}$ Potential Intensity (Maximum Velocity $\mathrm{m} \mathrm{s}^{-1}$ )

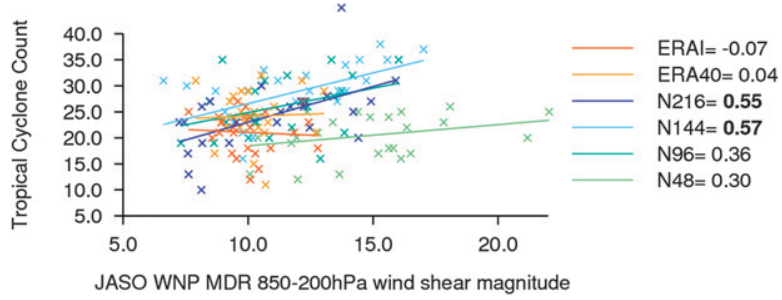

FIG. 13. As for Fig. 12 but for the northwestern Pacific.

varies interannually with storm count, seen most strongly for ERA-40; however, for the GCMs and reanalyses $850-\mathrm{hPa}$ absolute vorticity is the dominant environmental component. In the north Indian Ocean, the absolute vorticity is the dominant interannual variability component, with midlevel relative humidity showing some interannual variation with storm count. For the South Atlantic basin, relative humidity is the dominant factor varying interannually with storm count, as captured by the reanalyses and the higher resolution GCMs.

ERA-40 has higher midlevel relative humidity than seen in the GCMs or ERA-Interim; this may be a consequence of the problems with assimilation of humidity observations in ERA-40, as discussed in Bengtsson et al. (2004). The findings above, with the interannual variability of humidity correlating strongly with tropical cyclone numbers in several basins, suggest that this is the reason for the high number of storms tracked in ERA-40.

Analysis of the large-scale environmental parameters shows that in each tropical cyclone basin different environmental factors determine the interannual variability, which agrees with the findings of Camargo et al. (2007). GPI is a good indicator of interannual variability in the North Atlantic, where three out of four of its environmental components-PI, vertical wind shear, and low-level absolute vorticity-play a strong role in interannual variability. However, GPI may not be the best indicator of interannual variability of tropical cyclones in basins where certain environmental factors play a much stronger role in interannual variability than others, such as absolute vorticity in the northeast Pacific, with PI and wind shear parameters having little impact on interannual variability. In these basins the remaining parameters represented in GPI are important for tropical cyclogenesis and may be important on other time scales, but are not determining the interannual variability. When compared to reanalyses, higher resolution GCMs are more able to simulate the interannual variation of the environmental conditions that determine tropical cyclone variability, particularly in the North Atlantic and northwest Pacific basins. Improved understanding of the influence of largescale mechanisms on tropical cyclone activity may have the potential to lead to improved predictability of the interannual variability of tropical cyclone activity.

\section{Discussion and conclusions}

A systematic assessment of a hierarchy of global, high-resolution atmosphere-only GCMs, alongside high-resolution reanalyses and observational data, has been used to evaluate the role of spatial resolution on global tropical cyclone simulation. It is important to 
determine the ability of GCMs at these resolutions to simulate aspects of tropical cyclone behavior, as these GCMs, run for multicentury coupled simulations, are used to investigate the impact of long-term (e.g., interdecadal) climate variability on tropical cyclone activity. This will allow GCM simulations to supplement tropical cyclone observational data where the models show skill, allowing the climate modeling and impacts communities to gain a more comprehensive understanding of the varying location, frequency, and intensity of tropical cyclones. This has important implications for socioeconomic risk assessment.

The average annual number of tropical cyclones, both globally and in individual basins, particularly in the Northern Hemisphere, is simulated well for GCMs and reanalyses with $135-\mathrm{km}$ resolution and higher. Global distribution of the storms is well simulated by the AGCMs, particularly those with resolutions of $100 \mathrm{~km}$ or higher. Extracted storm tracks, however, have longer lifetimes than observed tracks: genesis is observed earlier, as a high relative vorticity center rather than at observed tropical storm stage; lysis may be identified later as the tracked storms are followed as they make extratropical transition into higher latitudes. This additional information is very useful for understanding the origin of tropical cyclones. The ability of a GCM to simulate the location of the tropical cyclones is important for the simulation of storm landfall. Landfall simulation has not been investigated in isolation in this study but is important to consider if GCMs are to be used for impact studies.

The GCMs with resolutions higher than $100 \mathrm{~km}$ show skill in the simulation of tropical cyclone interannual variability in the North Atlantic and North Pacific basins. This skill is assessed in terms of the ability of the GCMs to capture the interannual variability of the large-scale environmental variables known to influence tropical cyclogenesis, such as low-level absolute vorticity, potential intensity, vertical wind shear, and midtropospheric relative humidity. The GCMs capture the interannual variability of key large-scale parameters such as vertical wind shear, low-level absolute vorticity, and PI in the North Atlantic basin, and midlevel relative humidity and low-level vorticity in the North Pacific basins. However, the lower resolution AGCMs struggle to capture this variability. The importance of each environmental variable on tropical cyclone interannual variability depends on the basin.

AGCMs with observed SST forcing allow simulations to be directly comparable to each other and to tropical cyclone observations from the same period. However, it is important to consider the limitations of AGCMs, as using fixed SSTs neglect the negative feedback caused by the cooling effect of tropical cyclones on the ocean, which is known to distort tropical cyclone interannual variability (e.g., Waliser et al. 1999). This may be leading, for example, to the reduced interannual correlation between PI (which contains SST) and North Atlantic annual storm numbers in the GCM simulations. This can also have an impact on tropical cyclone intensities (Bender and Ginis 2000; Schade and Emanuel 1999).

Tropical cyclone intensity depends crucially on model resolution. The hierarchy of GCMs and reanalyses simulate tropical cyclones with higher intensities at higher resolution. However, at the resolutions considered in this study, convergence to observed tropical cyclone intensities is not achieved. When investigating the role of resolution in tropical cyclone climate projections using AGCM resolutions from 180 to $20 \mathrm{~km}$, Murakami and Sugi (2010) found that even with the finest resolution $(20 \mathrm{~km})$ tropical cyclone intensity was underestimated. It is acknowledged that GCMs, currently employed for multiyear simulations, are run at horizontal resolutions that are presently too coarse to simulate tropical cyclones at their full observed intensity, particularly around the inner core of the storms (Emanuel 2008), which may require resolution as fine as a few kilometers (Gentry and Lackmann 2010). It is assumed that the intensity and the frequency are effectively decoupled, as discussed in Zhao et al. (2009), so that GCM simulations of location and frequency may be considered reliable, despite resolution-based deficiencies in intensity simulation.

Despite inadequacy in intensity simulation, GCMs at resolutions higher than $100 \mathrm{~km}$ are shown to provide valuable insights into the effect of climate variability on storm activity in terms of tropical cyclone frequency, location, and interannual variability. In light of our findings, we recommend that GCMs be run at a resolution $100 \mathrm{~km}$ or higher for long-term model simulations (i.e., multidecadal to multicentury) used to investigate the impact of long-term climate variability on tropical cyclone behavior in terms of location and frequency. For intensity assessment, these resolutions can only provide an indication of relative change; for assessment of the absolute tropical cyclone intensities, very-high-resolution short-term simulations or dynamical-statistical downscaling approaches would need to be adopted. We are currently investigating methods in which to gain intensity information through dynamical-statistical bias correction. Zhao and Held (2010) show that, even with a simple statistical intensity adjustment to downscale simulated tropical cyclone winds from a 50-km GCM, they were able to gain intensity variability that corresponded much more closely with observed wind intensities.

Future papers on the analysis of our multicentury, fully coupled simulations will provide a platform to study long-term climate variability. The current study 
provides confidence in the atmospheric model component, as a basis for analyzing the fully coupled GCM simulations of tropical cyclone activity, for both current and future climate experiments.

Acknowledgments. This research was supported by a collaboration between the University of Reading and Willis. The models described were developed from the Met Office Hadley Centre Model by the U.K. HighResolution Modelling (HiGEM) Project and the U.K.Japan Climate Collaboration (UJCC). HiGEM is supported by a NERC High Resolution Climate Modelling Grant (R8/H12/123). UJCC was supported by the Foreign and Commonwealth Office Global Opportunities Fund, and this work is jointly funded by NERC and the Joint DECC/Defra Met Office Hadley Centre Climate Programme (GA01101). Model integrations were performed using the Japanese Earth Simulator supercomputer, supported by JAMSTEC. Thank you to the British Atmospheric Data Archive for hosting our data, ECMWF for providing ERA-40 and ERA-Interim, NASA for providing MERRA reanalyses, NOAA/National Climate Data Centre for providing IBTrACS data, and to Computational Modelling Services. We wish to thank Ming Zhao and Kevin Walsh for providing thorough reviews with very helpful comments and suggestions for improving the manuscript.

\section{REFERENCES}

Aiyyer, A., and C. Thorncroft, 2011: Interannual-to-multidecadal variability of vertical wind shear and tropical cyclone activity. J. Climate, 24, 2949-2462.

Bender, M. A., and I. Ginis, 2000: Real-case simulations of hurricaneocean interaction using a high-resolution coupled model: Effects on hurricane intensity. Mon. Wea. Rev., 128, 917-946.

Bengtsson, L., M. Botzet, and M. Esch, 1995: Hurricane-type vortices in a general circulation model. Tellus, 47A, 175-196.

-, K. I. Hodges, and S. Hagemann, 2004: Sensitivity of largescale atmospheric analyses to humidity observations and its impact on the global water cycle and tropical and extratropical weather systems in ERA40. Tellus, 56A, 202-217.

,-- , and M. Esch, 2007: Tropical cyclones in a T159 resolution global climate model: Comparison with observations and re-analysis. Tellus, 59A, 396-416.

Bister, M., and K. A. Emanuel, 1998: Dissipative heating and hurricane intensity. Meteor. Atmos. Phys., 52, 233-240.

Blender, R., and M. Schubert, 2000: Cyclone tracking in different spatial and temporal resolutions. Mon. Wea. Rev., 128, 377-384.

Broccoli, A. J., and S. Manabe, 1990: Can existing climate models be used to study anthropogenic changes in tropical cyclone climate? Geophys. Res. Lett., 17, 1917-1920.

Camargo, S. J., and A. H. Sobel, 2005: Western North Pacific tropical cyclone intensity and ENSO. J. Climate, 18, 2996-3006.

_, K. A. Emanuel, and A. H. Sobel, 2007: Use of a genesis potential index to diagnose ENSO effects on tropical cyclone genesis. J. Climate, 20, 4819-4834.
Chan, J. C. L., and K. S. Liu, 2004: Global warming and western North Pacific typhoon activity from an observational perspective. J. Climate, 17, 4590-4602.

Chia, H. H., and C. F. Ropelewski, 2002: The interannual variability of genesis location of tropical cyclones in the northwest Pacific. J. Climate, 15, 2934-2944.

Emanuel, K. A., 1995: Sensitivity of tropical cyclones to surface exchange coefficients and a revised steady-state model incorporating eye dynamics. J. Atmos. Sci., 52, 3969-3976.

, 2008: The hurricane-climate connection. Bull. Amer. Meteor. Soc., 87, 299-314.

, 2010: Stratospheric cooling and tropical cyclones. Preprints, 29th Conf. on Hurricanes and Tropical Meteorology, Tucson, AZ, Amer. Meteor. Soc., 4A.4. [Available online at http:// ams.confex.com/ams/pdfpapers/168302.pdf.]

- , and D. S. Nolan, 2004: Tropical cyclone activity and global climate. Preprints, 26th Conf. on Hurricanes and Tropical Meteorology, Miami, FL, Amer. Meteor. Soc., 240-241.

Evan, T. A., and S. J. Camargo, 2011: A climatology of Arabian Sea cyclonic storms. J. Climate, 24, 140-158.

Gentry, M. S., and G. M. Lackmann, 2010: Sensitivity of simulated tropical cyclone structure and intensity to horizontal resolution. Mon. Wea. Rev., 138, 688-704.

Godbole, R. V., 1977: The composite structure of the monsoon depression. Tellus, 29, 25-40.

Gray, W. M., 1979: Hurricanes: Their formation, structure, and likely role in the tropical circulation. Meteorology over the Tropical Oceans, D. B. Shaw, Ed., Royal Meteorological Society, $155-218$.

_ 1984: Atlantic seasonal hurricane frequency. Part I: El Niño and $30 \mathrm{mb}$ quasi-biennial oscillation influences. Mon. Wea. Rev., 112, 1649-1668.

Guilyardi, E., and Coauthors, 2004: Representing El Niño in coupled ocean-atmosphere GCMs: The dominant role of the atmospheric component. J. Climate, 17, 4623-4629.

Haarsma, R. J., J. F. B. Mitchell, and C. A. Senior, 1992: Tropical disturbances in a GCM. Climate Dyn., 8, 247-257.

Henderson-Sellers, A., and Coauthors, 1998: Tropical cyclones and global climate change: A post-IPCC assessment. Bull. Amer. Meteor. Soc., 79, 19-38.

Hodges, K. I., 1995: Feature tracking on a unit sphere. Mon. Wea. Rev., 123, 3458-3465. 1996: Spherical nonparametric estimators applied to the UGAMP model integration for AMIP. Mon. Wea. Rev., 124, 2914-2932.

, 1999: Adaptive constraints for feature tracking. Mon. Wea. Rev., 127, 1362-1373.

Holland, G., and P. Webster, 2007: Heightened tropical cyclone activity in the North Atlantic: Natural variability or climate trend? Philos. Trans. Roy. Soc., 365, 2695-2716.

Hoskins, B. J., and K. I. Hodges, 2002: New perspectives on the Northern Hemisphere winter storm tracks. J. Atmos. Sci., 59, 1041-1061.

Johns, T. C., and Coauthors, 2006: The new Hadley Centre climate model (HadGEM1): Evaluation of coupled simulations. J. Climate, 19, 1327-1353.

Knapp, K. R., M. C. Kruk, D. H. Levinson, H. J. Diamond, and C. J. Neumann, 2010: The International Best Track Archive for Climate Stewardship (IBTrACS): Unifying tropical cyclone best track data. Bull. Amer. Meteor. Soc., 91, 363-376.

Kobayashi, C., and M. Sugi, 2004: Impact of horizontal resolution on the simulation of the Asian summer monsoon and tropical cyclones in the JMA global model. Climate Dyn., 23, 165-176. 
Landsea, C. W., 1993: A climatology of intense (or major) Atlantic hurricanes. Mon. Wea. Rev., 121, 1703-1713.

- 2007: Counting Atlantic tropical cyclones back to 1900. Eos, Trans. Amer. Geophys. Union, 88, 197-208.

_ B. A. Harper, K. Hoarau, and J. A. Knaff, 2006: Can we detect trends in extreme tropical cyclones? Science, 28, 452-454.

LaRow, T. E., Y. K. Lim, D. W. Shin, E. P. Chassignet, and S. Cooke, 2008: Atlantic basin seasonal hurricane simulations. J. Climate, 21, 3191-3206.

Manabe, S., J. L. Holloway, and H. M. Stone, 1970: Tropical circulation in a time-integration of a global model of the atmosphere. J. Atmos. Sci., 27, 580-613.

Manganello, J. V., and Coauthors, 2012: Tropical cyclone climatology in a 10-km global atmospheric GCM: Toward weatherresolving climate modeling. J. Climate, 25, 3867-3893.

Martin, G. M., M. A. Ringer, V. D. Pope, A. Jones, C. Dearden, and T. J. Hinton, 2006: The physical properties of the atmosphere in the new Hadley Centre Global Environmental Model (HadGEM1). Part I: Model description and global climatology. J. Climate, 19, 1274-1301.

Murakami, H., and M. Sugi, 2010: Effect of model resolution on tropical cyclone climate projections. SOLA, 6, 73-76.

$\ldots$, and B. Wang, 2010: Future change of North Atlantic tropical cyclone tracks: Projection by a $20-\mathrm{km}-\mathrm{mesh}$ global atmospheric model. J. Climate, 23, 2699-2721.

Oouchi, K., J. Yoshimura, H. Yoshimura, R. Mizuta, S. Kusunoki, and A. Noda, 2006: Tropical cyclone climatology in a globalwarming climate as simulated in a $20 \mathrm{~km}$-mesh global atmospheric model: Frequency and wind intensity analysis. J. Meteor. Soc. Japan, 84, 259-276.

Pielke, R. A., Jr., and R. A. Pielke Sr., 1997: Hurricanes: Their Nature and Impacts on Society. Wiley, 279 pp.

Ren, F., J. Liang, G. Wu, W. Dong, and X. Yang, 2011: Reliability analysis of climate change of tropical cyclone activity over the western North Pacific. J. Climate, 24, 5887-5898.

Rienecker, M. M., and Coauthors, 2011: MERRA: NASA's modern-era retrospective analysis for research and applications. J. Climate, 24, 3624-3648.

Ringer, M. A., and Coauthors, 2006: The physical properties of the atmosphere in the new Hadley Centre Global Environmental Model (HadGEM1). Part II: Aspects of variability and regional climate. J. Climate, 19, 1302-1326.

Roberts, M. J., and Coauthors, 2009: Impact of resolution in the tropical Pacific circulation in a matrix of coupled models. J. Climate, 22, 2541-2556.

Schade, L. R., and K. A. Emanuel, 1999: The ocean's effect on the intensity of tropical cyclones: Results from a simple coupled atmosphere-ocean model. J. Atmos. Sci., 56, 642-651.

Shaffrey, L. C., and Coauthors, 2009: U.K. HiGEM: The new U.K. high-resolution global environment model-model description and basic evaluation. J. Climate, 22, 1861-1896.
Simmons, A., S. Uppala, D. Dee, and S. Kobayashi, 2007: ERAInterim: New ECMWF reanalysis products from 1989 onwards. ECMWF Newsletter, No. 110, ECMWF, Reading, United Kingdom, 25-35.

Solomon, S., D. Qin, M. Manning, M. Marquis, K. Averyt, M. M. B. Tignor, H. L. Miller, Jr., and Z. Chen, Eds., 2007: Climate Change 2007: The Physical Science Basis. Cambridge University Press, $996 \mathrm{pp}$.

Southern, R., 1979: The global socio-economic impact of tropical cyclones. Aust. Meteor. Mag., 27, 175-195.

Taylor, K., D. Williamson, and F. Zwiers, 2000: The sea surface temperature and sea-ice concentration boundary conditions for AMIP II simulations. PCMDI Tech. Rep. 60, 28 pp.

Uppala, S. M., and Coauthors, 2005: The ERA-40 Re-Analysis. Quart. J. Roy. Meteor. Soc., 131, 2961-3012.

Villarini, G., G. A. Vecchi, and J. A. Smith, 2010: Modeling the dependence of tropical storm counts in the North Atlantic basin on climate indices. Mon. Wea. Rev., 138, 2681-2705.

Vitart, F., and T. N. Stockdale, 2001: Seasonal forecasting of tropical storms using coupled GCM integrations. Mon. Wea. Rev., 129, 2521-2537.

Waliser, D. E., K. M. Lau, and J. H. Kim, 1999: The influence of coupled sea surface temperatures on the Madden-Julian oscillation: A model perturbation experiment. J. Atmos. Sci., 56, 333-358.

Walsh, K. J. E., M. Fiorino, C. W. Landsea, and K. L. McInnes, 2007: Objectively determined resolution-dependent threshold criteria for the detection of tropical cyclones in climate models and reanalysis. J. Climate, 20, 2307-2314.

_ S. Lavender, E. Scoccimarro, and H. Murakami, 2012: Resolution dependence of tropical cyclone formation in CMIP3 and finer resolution models. Climate Dyn., doi:10.1007/ s00382-012-1298-z, in press.

Zehnder, J. A., D. M. Powell, and D. L. Ropp, 1999: The interaction of easterly waves, orography, and the intertropical convergence zone in the genesis of eastern Pacific tropical cyclones. Mon. Wea. Rev., 127, 1566-1585.

Zhao, M., and I. M. Held, 2010: An analysis of the effect of global warming on the intensity of Atlantic hurricanes using a GCM with statistical refinement. J. Climate, 23, 6382-6393.

$\longrightarrow$, and — 2012: TC-permitting GCM simulations of hurricane frequency response to sea surface temperature anomalies projected for the late-twenty-first century. J. Climate, 25, 29953009 .

$\ldots,-$, S.-J. Lin, and G. A. Vecchi, 2009: Simulations of global hurricane climatology, interannual variability, and response to global warming using a 50-km-resolution GCM. J. Climate, 22, 6653-6678.

,-- , and $—$ 2012: Some counterintuitive dependencies of tropical cyclone frequency on parameters in a GCM. J. Atmos. Sci., 69, 2272-2283. 